\title{
A Generalized Labeled Multi-Bernoulli Filter with Object Spawning
}

\author{
Daniel S. Bryant, Ba-Tuong Vo, Ba-Ngu Vo, and Brandon A. Jones
}

\begin{abstract}
Previous labeled random finite set filter developments use a motion model that only accounts for survival and birth. While such a model provides the means for a multi-object tracking filter such as the Generalized Labeled Multi-Bernoulli (GLMB) filter to capture object births and deaths in a wide variety of applications, it lacks the capability to capture spawned tracks and their lineages. In this paper, we propose a new GLMB based filter that formally incorporates spawning, in addition to birth. This formulation enables the joint estimation of a spawned object's state and information regarding its lineage. Simulations results demonstrate the efficacy of the proposed formulation.
\end{abstract}

Index Terms-Random finite sets, generalized labeled multiBernoulli filter, object spawning, multi-target tracking, multiobject filtering, Bayesian estimation.

\section{INTRODUCTION}

Multi-object tracking is concerned with estimating the number of objects and their trajectories in the presence of object appearance/disappearance, clutter, and uncertainties in detection, measurements, and data associations. The field covers a wide variety of applications which include aviation [1], astrodynamics, [2]-[4], defense [5], robotics [6], [7], and cell biology [8], [9]. Three of the most prominent approaches to multi-object filtering are Multiple Hypotheses Tracking (MHT) [10]-[13], Joint Probabilistic Data Association (JPDA) [14], and random finite sets (RFSs) [15], [16].

In a multi-object system, new objects appear either from spontaneous birth or spawning from existing objects. While many existing multi-object tracking approaches can accommodate spontaneous birth, so far only the RFS approach offers a principled treatment of spawning in terms of modeling and estimation [17], [15]. Central to the RFS approach is the multi-object Bayes recursion [15] which recursively propagates the multi-object posterior forward in time. The first order approximation more commonly known as the Probability Hypothesis Density (PHD) filter accounts for both birth and spawning [17]. However, its generalization, the Cardinalized PHD (CPHD) filter, was derived without a spawning model [18]. The CPHD filter with spawning is generally intractable and approximations were derived in [19], [20].

D. S. Bryant is with the Department of Aerospace Engineering Sciences, University of Colorado Boulder, Boulder, CO 80309 USA (e-mail: daniel.bryant@colorado.edu).

B.-T. Vo and B.-N. Vo are with the Department of Electrical and Computer Engineering, Curtin University, Bentley, WA 6102, Australia (e-mail: batuong.vo@curtin.edu.au; ba-ngu.vo@curtin.edu.au).

B. A. Jones is with the Department of Aerospace Engineering and Engineering Mechanics, The University of Texas Austin, Austin, TX, 78712 USA (e-mail: brandon.jones@utexas.edu).
Information on lineage or ancestry is an important aspect of tracking multiple spawning objects. For example, in (biological) cell tracking, information on a cell's lineage is important to the analyis of cell behavior [8], [21]-[23]. For space situational awareness, information on the ancestry of debris is important to the analysis of fragmentation events [24][26]; moreover, country of origin and launch site, information required to add a space object to the United States Strategic Command (USSTRATCOM) catalog [27], can be derived from ancestry information. Even with spawning models, the PHD/CPHD filters [17], [19], [20] only provide estimates of spawned objects' states, but no information on their ancestries. Further, in applications where ancestry information is not required, it is also possible to estimate spawned objects using RFS-based multi-object filters with measurement-driven (spontaneous) birth models [28], [29]. Hence, a complete treatment of modeling and estimation for spawning objects should address the issue of ancestry.

Labeled RFSs enable ancestry information to be incorporated into the modeling and estimation of spawning objects. Approximate multi-object Bayes filters such as the PHD [17], CPHD [18], and multi-Bernoulli [15], [30], [31] filters were not formulated to estimate tracks, without which the ancestries of the objects are, conceptually, not traceable. On the other hand, labeled RFSs provide the means for identifying and estimating individual object tracks [32], thereby making it possible, conceptually, to trace their ancestors. Furthermore, as demonstrated in this paper, the labels used to identify individual tracks can also be encoded with ancestry information, which can be assimilated by RFS spawning models, and subsequently inferred from the labels obtained using labeled RFS estimation techniques.

Under the labeled RFS formulation, the multi-object Bayes recursion (without spawning) admits an analytic solution known as the Generalized Labeled Multi-Bernoulli (GLMB) filter [32], [33], which can be implemented with linear complexity in the number of measurements and quadratic in the number of hypothesized tracks [34]. This on-line multi-object tracker is based on the GLMB family of conjugate priors that enjoys a number of nice analytical properties, e.g., the void probability functional-a necessary and sufficient statistic-of a GLMB, the Cauchy-Schwarz divergence between two GLMBs [35], the $L_{1}$-distance between a GLMB and its truncation, can all be computed in closed form [33]. Of direct relevance to this work is the fact that the GLMB family is flexible enough to approximate any labeled RFS density with matching intensity function and cardinality distribution [36].

In this paper, we propose a new GLMB based filter that 
formally incorporates spawning, in addition to birth. Using labeled RFSs we encode ancestry information into the labels of individual object states and propose a labeled RFS spawning model. When a track is instantiated by spontaneous birth, its label contains information pertaining to when an object is born and from which birth region [32]. Similarly, for a track instantiated by spawning, its label contains information pertaining to when and from which parent it originated. Under such a spawning model, the multi-object prediction and filtering densities are no longer GLMBs, even if the initial prior is a GLMB. To derive a tractable filter, following [34] we combine the prediction and update into a single step and approximate the labeled multi-object filtering density by a GLMB with matching first moment and cardinality using the technique in [36]. The result is a recursion that propagates the GLMB approximation of the labeled multi-object filtering density, from which the states of the spawned objects and their labels, hence lineage, can be jointly inferred.

The remainder of this paper is arranged as follows: In Section II background is provided on the GLMB filter, its use in approximation of general labeled RFS densities, and its efficient implementation. In Section III the derivation, approximation, and joint prediction and update of object spawning inclusive GLMB densities is developed. Simulation results are presented in Section IV and in Section V concluding remarks are given.

\section{BACKGROUND}

This section provides background on GLMB filter implementation pertinent to the formulation of our multi-object filtering problem.

\section{A. Labeled Random Finite Sets}

Instead of a single system state $x$ in the state space $\mathbb{X}$, in a multi-object system we consider a finite set $X \subset \mathbb{X}$ as the multi-object state. Further, in a Bayesian framework the multi-object state is modeled as an RFS, i.e., a finite-setvalued random variable [17]. An RFS, also known as a simple finite point process, consists of a random number of points that are, themselves, random and unordered. An RFS can be described by the multi-object density-defined to be the set derivative of its belief functional [15]-shown to be equivalent to a probability density in [37.

A labeled RFS is a marked simple finite point process with state space $\mathbb{X}$ and discrete mark space $\mathbb{L}$, such that each realization has distinct marks [32], [33]. The distinct marks or labels provide the means to identify trajectories or tracks of individual objects since a trajectory is a time-sequence of states with the same label. Let $\mathcal{L}: \mathbb{X} \times \mathbb{L} \rightarrow \mathbb{L}$ be the projection $\mathcal{L}((x, \ell))=\ell$, then the labels of realization $\mathbf{X} \subset \mathbb{X} \times \mathbb{L}$ are then $\mathcal{L}(\mathbf{X})=\{\mathcal{L}(\mathbf{x}): \mathbf{x} \in \mathbf{X}\}$. The realization $\mathbf{X}$ is said to have distinct labels if and only if it has the the same cardinality as its labels $\mathcal{L}(\mathbf{X})$. This concept is compactly formulated by the distinct label indicator defined by [32], [33]

$$
\Delta(\mathbf{X})=\delta_{|\mathbf{X}|}(|\mathcal{L}(\mathbf{X})|)
$$

where $|X|$ denotes the cardinality of a finite set $X$, and

$$
\delta_{Y}(X) \triangleq \begin{cases}1, & \text { if } X=Y \\ 0, & \text { otherwise }\end{cases}
$$

denotes a generalization of the Kroneker delta that takes arbitrary arguments such as integers, sets, vectors etc.

Throughout this paper we adhere to the convention that lower case letters represent single-object states, e.g., $x, \mathbf{x}$, while upper case letters represent multi-object states, e.g., $X, \mathbf{X}$. Bold symbols represent labeled states and their distributions/statistics, e.g., $\mathbf{x}, \mathbf{X}, \boldsymbol{\pi}$, etc., to distinguish them from unlabeled ones. Blackboard letters represent spaces, e.g., $\mathbb{X}, \mathbb{Z}, \mathbb{L}, \mathbb{N}$. We use the standard inner product notation $\langle f, g\rangle \triangleq \int f(x) g(x) d x$, the multi-object exponential notation $h^{X} \triangleq \prod_{x \in X} h(x)$, where $h$ is a real-valued function and $h^{\emptyset}=1$ by convention, and the inclusion function notation, a generalization of the indicator function, by

$$
1_{Y}(X) \triangleq \begin{cases}1, & \text { if } X \subseteq Y \\ 0, & \text { otherwise }\end{cases}
$$

Additionally, the list of variables $X_{m}, X_{m+1}, \ldots, X_{n}$ is abbreviated as $X_{m: n}$ and where it is convenient we let the symbol + denote the time index at the next time and its absence denote the time index at the current time, e.g., the state $x_{k}$ at the current time and the state $x_{k+1}$ at the next time can equivalently be denoted as $x$ and $x_{+}$, respectively.

\section{B. Generalized Labeled Multi-Bernoulli}

A GLMB is a labeled RFS with state space $\mathbb{X}$ and label space $\mathbb{L}$ distributed according to [32], [33]

$$
\boldsymbol{\pi}(\mathbf{X})=\Delta(\mathbf{X}) \sum_{(I, \xi) \in \mathcal{F}(\mathbb{L}) \times \Xi} w^{(I, \xi)} \delta_{I}(\mathcal{L}(\mathbf{X}))\left[p^{(\xi)}\right]^{\mathbf{X}}
$$

where $\Xi$ is a given discrete space, each $p^{(\xi)}(\cdot, \ell)$ is a (singleobject) probability density on $\mathbb{X}$ (i.e., $\int p^{(\xi)}(x, \ell) d x=1$ with each $x \in \mathbb{X}$ denoting a single-object state and each $\ell \in \mathbb{L}$ denoting a distinct label), and each $w^{(I, \xi)}$ is non-negative such that

$$
\sum_{I \in \mathcal{F}(\mathbb{L})} \sum_{\xi \in \Xi} w^{(I, \xi)}(L)=1 .
$$

Each GLMB density component $(I, \xi)$ in (1) consists of a weight $w^{(I, \xi)}$ that depends solely on the labels of the multiobject state $\mathbf{X}$ and the multi-object exponential $\left[p^{(\xi)}\right]^{\mathbf{X}}$, which is a product of single-object probability densities.

Also relevant to this work is the Labeled Multi-Bernoulli (LMB). An LMB $\mathbf{X}$ defined on $\mathbb{X} \times \mathbb{L}$ is an RFS with parameter set $\left\{\left(r^{(\zeta)}, p^{(\zeta)}\right): \zeta \in \Psi\right\}$ distributed according to [32

$$
\boldsymbol{\pi}(\mathbf{X})=\Delta(\mathbf{X}) 1_{\alpha(\Psi)}(\mathcal{L}(\mathbf{X}))[\Phi(\mathbf{X}, \cdot)]^{\Psi}
$$

where $\alpha: \Psi \rightarrow \mathbb{L}$ is a 1-1 mapping (usually an identity mapping) and

$$
\begin{aligned}
\Phi(\mathbf{X}, \zeta)=\sum_{(x, \ell) \in \mathbf{X}} \delta_{\alpha(\zeta)}(\ell) r^{(\zeta)} p^{(\zeta)}(x) \\
\quad+\left(1-1_{\mathcal{L}(\mathbf{X})}(\alpha(\zeta))\right)\left(1-r^{(\zeta)}\right) .
\end{aligned}
$$




\section{Multi-object Bayes Filter}

Using the convention detailed in [32], a label $\ell=(t, i)$ in the space $\mathbb{L}$ of labels at the current time $k$ is an ordered pair, where the first term $t \leq k$ denotes time of birth, and the second term $i \in \mathbb{N}$ is a unique index distinguishing objects born at the same time. Birth labels at the next time belong to the space $\mathbb{B}_{+}=\{(k+1, i): i \in \mathbb{N}\}$, hence $\mathbb{L} \cap \mathbb{B}_{+}=\emptyset$ and the label space at the next time is $\mathbb{L}_{+}=\mathbb{L} \cup \mathbb{B}_{+}$.

The history $\mathbf{X}_{0: k}$ of labeled multi-object states contains the set of all trajectories up to time $k$. All information on the set of trajectories conditioned on the observation history $Z_{1: k}$, is captured in the multi-object posterior density $\pi_{0: k}\left(\cdot \mid Z_{1: k}\right)$, which incorporates the evolution of the multi-object state via the multi-object transition density, as well as the observed data via the multi-object likelihood [15].

1) Multi-Object Transition Model: The multi-object transition density $\mathbf{f}_{+}(\cdot \mid \mathbf{X})$ models the evolution of a given multiobject state $\mathbf{X}$ to the next time and encapsulates all information pertaining to loss of objects via thinning, movement of surviving objects via Markov shifts, and appearance of new objects via superposition.

Given a single-object state $\mathbf{x} \in \mathbf{X}$ at the current time, an object either survives to the next time with probability $p_{\mathrm{S}}(x, \ell)$ and moves to a new state $\left(x_{+}, \ell_{+}\right)$with probability density $f_{\mathrm{S},+}\left(x_{+} \mid x, \ell\right) \delta_{\ell}\left(\ell_{+}\right)$, or dies with probability $q_{\mathrm{S}}(x, \ell)=1-p_{\mathrm{S}}(x, \ell)$. Assuming that, conditional on $\mathbf{X}$, the transition of kinematic states $\mathbf{x} \in \mathbf{X}$ are mutually independent, we model the set $\mathbf{X}_{\mathrm{S},+}$ of surviving objects at the next time as a conditional LMB RFS distributed according to [32]

$$
\begin{aligned}
& \mathbf{f}_{\mathrm{S},+}\left(\mathbf{X}_{\mathrm{S},+} \mid \mathbf{X}\right)= \\
& \quad \Delta(\mathbf{X}) \Delta\left(\mathbf{X}_{\mathrm{S},+}\right) 1_{\mathcal{L}(\mathbf{X})}\left(\mathcal{L}\left(\mathbf{X}_{\mathrm{S},+}\right)\right)\left[\Phi_{\mathrm{S},+}\left(\mathbf{X}_{\mathrm{S},+} \mid \cdot\right)\right]^{\mathbf{X}}
\end{aligned}
$$

where

$$
\begin{aligned}
\Phi_{\mathrm{S},+}\left(\mathbf{X}_{\mathrm{S},+} \mid x, \ell\right)=\sum_{\left(x_{+}, \ell_{+}\right) \in \mathbf{X}_{\mathrm{S},+}} & \delta_{\ell}\left(\ell_{+}\right) p_{\mathrm{S}}(x, \ell) f_{\mathrm{S},+}\left(x_{+} \mid x, \ell\right) \\
+ & {\left[1-1_{\mathcal{L}\left(\mathbf{X}_{\mathrm{S},+}\right)}(\ell)\right] q_{\mathrm{S}}(x, \ell) . }
\end{aligned}
$$

A new object with state $\left(x_{+}, \ell_{+}\right)$appears at the next time with probability $r_{\mathrm{B},+}\left(\ell_{+}\right)$and probability density $p_{\mathrm{B},+}\left(x_{+}, \ell_{+}\right)$, or does not with probability $1-r_{\mathrm{B},+}\left(\ell_{+}\right)$. Modeling object birth as an LMB RFS, the set $\mathbf{X}_{\mathrm{B},+}$ of new objects born at the next time is distributed according to [32]

$$
\mathbf{f}_{\mathrm{B},+}\left(\mathbf{X}_{\mathrm{B},+}\right)=\Delta\left(\mathbf{X}_{\mathrm{B},+}\right) w_{\mathrm{B},+}\left(\mathcal{L}\left(\mathbf{X}_{\mathrm{B},+}\right)\right)\left[p_{\mathrm{B},+}\right]^{\mathbf{X}_{\mathrm{B},+}}
$$

where $w_{\mathrm{B},+}(L)=1_{\mathbb{B}_{+}(L)}\left[1-r_{\mathrm{B},+}\right]^{\mathbb{B}_{+}-L}\left[r_{\mathrm{B},+}\right]^{L}$.

The multi-object state at the next time is the superposition of birth and surviving objects, i.e., $\mathbf{X}_{+}=\mathbf{X}_{\mathrm{S},+} \cup \mathbf{X}_{\mathrm{B},+}$, and since $\mathbb{L} \cap \mathbb{B}_{+}=\emptyset$, labeled birth and surviving objects are mutually independent. Thus, the multi-object transition kernel ultimately reduces to the product of birth and survival transition densities [32]

$$
\mathbf{f}_{+}\left(\mathbf{X}_{+} \mid \mathbf{X}\right)=\mathbf{f}_{\mathrm{S},+}\left(\mathbf{X}_{+} \cap(\mathbb{X} \times \mathbb{L}) \mid \mathbf{X}\right) \mathbf{f}_{\mathrm{B},+}\left(\mathbf{X}_{+}-(\mathbb{X} \times \mathbb{L})\right),
$$

where $\mathbf{X}_{+} \cap(\mathbb{X} \times \mathbb{L})$ is the subset of $\mathbf{X}_{+}$consisting of surviving objects.
2) Multi-object Measurement Model: The multi-object likelihood is a multi-object density $g(\cdot \mid \mathbf{X})$ that models the multi-object observation generated by a given multi-object state $\mathbf{X}$, and encapsulates all information pertaining to missed detections via thinning, detections (observations of detected objects) via Markov shifts and clutter (false observations) via superposition.

The multi-object observation $Z=\left\{z_{1}, \ldots, z_{|Z|}\right\}$ is the superposition of detections and clutter. Each state $(x, \ell) \in \mathbf{X}$ is either detected with probability $p_{\mathrm{D}}(x, \ell)$ and generates an observation $z \in Z$ with likelihood $g(z \mid x, \ell)$ or missed with probability $q_{\mathrm{D}}(x, \ell)=1-p_{\mathrm{D}}(x, \ell)$. The multi-object likelihood is given by [32], [33]

$$
g(Z \mid \mathbf{X}) \propto \sum_{\theta \in \Theta(\mathcal{L}(\mathbf{X}))} \prod_{(x, \ell) \in \mathbf{X}} \psi^{(\theta(\ell))}(x, \ell \mid Z)
$$

where

$$
\begin{aligned}
\psi^{(j)}\left(x, \ell \mid\left\{Z_{1:|Z|}\right\}\right)= & \delta_{0}(j) q_{\mathrm{D}}(x, \ell) \\
& +\left(1-\delta_{0}(j)\right) \frac{p_{\mathrm{D}}(x, \ell) g\left(z_{j} \mid x, \ell\right)}{\kappa\left(z_{j}\right)},
\end{aligned}
$$

$\kappa(\cdot)$ is the intensity of Poisson clutter, and $\Theta(L)$ denotes the space of mappings $\theta: L \rightarrow\{0:|Z|\}$ that are 1-1 when restricting the range to the positive integers, i.e., $\theta(i)=\theta(j)>$ 0 implies $i=j$.

3) Multi-object Bayes recursion: While the multi-object posterior density can be approximated by Markov Chain Monte Carlo [38], [39], these techniques are still expensive and not suitable for on-line applications. For real-time tracking, a more tractable alternative is the marginal $\boldsymbol{\pi}(\cdot) \triangleq \boldsymbol{\pi}_{k}\left(\cdot \mid Z_{1: k}\right)$ called the multi-object filtering density, which can be recursively propagated by the multi-object Bayes filter [17], [15]

$$
\boldsymbol{\pi}_{+}\left(\mathbf{X}_{+}\right) \propto g_{+}\left(Z_{+} \mid \mathbf{X}_{+}\right) \int \mathbf{f}_{+}\left(\mathbf{X}_{+} \mid \mathbf{X}\right) \boldsymbol{\pi}(\mathbf{X}) \delta \mathbf{X},
$$

where the integral is a set integral defined for any function $\mathbf{f}: \mathcal{F}\left(\mathbb{X} \times \mathbb{L}_{k}\right) \rightarrow \mathbb{R}$ by

$$
\int \mathbf{f}(\mathbf{X}) \delta \mathbf{X}=\sum_{i=0}^{\infty} \frac{1}{i !} \int \mathbf{f}\left(\left\{\mathbf{x}_{1}, \ldots, \mathbf{x}_{i}\right\}\right) d\left(\mathbf{x}_{1}, \ldots, \mathbf{x}_{i}\right) .
$$

Note that Bayes optimal multi-object estimators can be formulated by minimizing the Bayes risk, e.g., the marginal multiobject estimator [15].

An analytic solution to the labeled Bayes multi-object filter (9), known as the Generalized Labeled Multi-Bernoulli (GLMB) filter, was derived in [32], while a particle approximation was implemented in [40] using the generic multi-object particle algorithm proposed in [37].

\section{Fast GLMB Filter Implementation}

The first GLMB filter implementation consists of prediction and update stages, each requiring independent truncations of GLMB densities [32], [33]. Alternatively, a substantially more efficient implementation of the GLMB filter [34], hereafter referred to as the fast GLMB implementation, employs a single joint prediction/update stage requiring only one truncation procedure. This work employs the fast GLMB implementation, 
thus for convenience, we introduce pertinent expressions and conventions for GLMB joint prediction/update and formulation of the GLMB truncation problem originally presented in [34]. We expand on this material in Section III-D to incorporate spawning.

Given the GLMB filtering density (1) at the current time, the GLMB filtering density at the next time is given by 34$]^{1}$

$$
\begin{aligned}
\boldsymbol{\pi}_{+}\left(\mathbf{X}_{+}\right) \propto & \Delta\left(\mathbf{X}_{+}\right) \sum_{I, \xi, I_{+}, \theta_{+}} w^{(I, \xi)} w^{\left(I, \xi, I_{+}, \theta_{+}\right)}\left(Z_{+}\right) \\
& \times \delta_{I_{+}}\left(\mathcal{L}\left(\mathbf{X}_{+}\right)\right)\left[p_{+}^{\left(\xi, \theta_{+}\right)}\left(\cdot \mid Z_{+}\right)\right]^{\mathbf{X}_{+}}
\end{aligned}
$$

where $I \in \mathcal{F}\left(\mathbb{L}_{)}\right), \xi \in \Xi, I_{+} \in \mathcal{F}\left(\mathbb{L}_{+}\right), \theta_{+} \in \Theta_{+}\left(I_{+}\right)$, and

$$
\begin{aligned}
& w^{\left(I, \xi, I_{+}, \theta_{+}\right)}\left(Z_{+}\right)= {\left[1-\bar{p}_{\mathrm{S}}^{(\xi)}\right]^{I-I_{+}}\left[\bar{p}_{\mathrm{S}}^{(\xi)}\right]^{I \cap I_{+}} } \\
& \times\left[1-r_{\mathrm{B},+}\right]^{\mathbb{B}_{+}-I_{+}}\left[r_{\mathrm{B},+}\right]^{\mathbb{B}_{+} \cap I_{+}} \\
& \times\left[\bar{\psi}_{+}^{\left(\xi, \theta_{+}\right)}\left(\cdot \mid Z_{+}\right)\right]^{I_{+}}, \\
& \bar{p}_{\mathrm{S}}^{(\xi)}(\ell)=\left\langle p_{\mathrm{S}}(\cdot, \ell), p^{(\xi)}(\cdot, \ell)\right\rangle \\
& \bar{\psi}_{+}^{\left(\xi, \theta_{+}\right)}\left(\ell_{+} \mid Z_{+}\right)=\left\langle\bar{p}_{+}^{(\xi)}\left(\cdot, \ell_{+}\right), \psi_{+}^{\left(\theta_{+}\left(\ell_{+}\right)\right)}\left(\cdot, \ell_{+} \mid Z_{+}\right)\right\rangle, \\
& p_{+}^{\left(\xi, \theta_{+}\right)}\left(x_{+}, \ell_{+} \mid Z_{+}\right)= \frac{\bar{p}_{+}^{(\xi)}\left(x_{+}, \ell_{+}\right) \psi_{+}^{\left(\theta_{+}\left(\ell_{+}\right)\right)}\left(x_{+}, \ell_{+} \mid Z_{+}\right)}{\bar{\psi}_{+}^{\left(\xi, \theta_{+}\right)}\left(\ell_{+} \mid Z_{+}\right)} \\
& \bar{p}_{+}^{(\xi)}\left(x_{+}, \ell_{+}\right)= 1_{\mathbb{B}_{+}}\left(\ell_{+}\right) p_{\mathrm{B},+}\left(x_{+}, \ell_{+}\right)+1_{\mathbb{L}}\left(\ell_{+}\right) \\
& \times \frac{\left\langle p_{\mathrm{S}}\left(\cdot, \ell_{+}\right) f_{\mathrm{S},+}\left(x_{+} \mid \cdot, \ell_{+}\right), p^{(\xi)}\left(\cdot, \ell_{+}\right)\right\rangle}{\bar{p}_{\mathrm{S}}^{(\xi)}\left(\ell_{+}\right)} .
\end{aligned}
$$

Though 10 is not strictly GLMB, it does take on GLMB form when rewritten as a sum over $I_{+}, \xi, \theta_{+}$with weights [34]

$$
w_{+}^{\left(I_{+}, \xi, \theta_{+}\right)}\left(Z_{+}\right) \propto \sum_{I} w^{(I, \xi)} w^{\left(I, \xi, I_{+}, \theta_{+}\right)}\left(Z_{+}\right) .
$$

Efficient implementation of the GLMB recursion 10$]$ is achieved by propagating only the components with significant $w^{\left(I, \xi, I_{+}, \theta_{+}\right)}\left(Z_{+}\right)$through time, i.e., for each component $(I, \xi)$ from the GLMB density at the current time and a multiobject observation $Z_{+}$at the next time, the set of pairs $\left(I_{+}, \theta_{+}\right) \in \mathcal{F}(\mathbb{L}) \times \Theta_{+}\left(I_{+}\right)$with significant $w^{\left(I, \xi, I_{+}, \theta_{+}\right)}\left(Z_{+}\right)$ are retained while the rest are discarded. The truncation procedure is described as follows.

Consider a fixed component $(I, \xi)$, and enumerate $Z_{+}=$ $\left\{z_{1:\left|Z_{+}\right|}\right\}, \mathbb{B}_{+}=\left\{\ell_{1: K}\right\}$, and $I=\left\{\ell_{K+1: P}\right\}$. For each pair $\left(I_{+}, \theta_{+}\right) \in \mathcal{F}(\mathbb{L}) \times \Theta_{+}\left(I_{+}\right)$, an equivalent $P$-dimensional vector representation $\gamma=\left(\gamma_{1: P}\right) \in\left\{-1:\left|Z_{+}\right|\right\}^{P}$ is defined as

$$
\gamma_{i}= \begin{cases}\theta_{+}\left(\ell_{i}\right), & \text { if } \ell_{i} \in I_{+} \\ -1, & \text { otherwise }\end{cases}
$$

Note that $\gamma$ inherits the positive 1-1 property from $\theta_{+}$, and that $I_{+}$and $\theta_{+}: I_{+} \rightarrow\left\{0:\left|Z_{+}\right|\right\}$can be recovered by

$$
I_{+}=\left\{\ell_{i} \in \mathbb{B}_{+} \cup I: \gamma_{i} \geq 0\right\}, \quad \theta_{+}\left(\ell_{i}\right)=\gamma_{i} .
$$

${ }^{1}$ In the interest of simplifying notation, note that $\sum_{(I, \xi) \in \mathcal{F}(\mathbb{L}) \times \Xi} a^{(I, \xi)}=$ $\sum_{I, \xi} a^{(I, \xi)}$ when the definitions $I \in \mathcal{F}(\mathbb{L})$ and $\xi \in \Xi$ are provided.
Assuming that, for all $i \in\{1: P\}, \bar{p}_{\mathrm{S}}^{(\xi)}\left(\ell_{i}\right) \in(0,1)$ and $\bar{p}_{\mathrm{D},+}^{(\xi)}\left(\ell_{i}\right) \triangleq\left\langle p_{\mathrm{D},+}\left(\cdot, \ell_{i}\right), \bar{p}_{+}^{(\xi)}\left(\cdot, \ell_{i}\right)\right\rangle \in(0,1)$, we define for each $j \in\left\{-1:\left|Z_{+}\right|\right\}$

$$
\eta_{i}(j)= \begin{cases}1-r_{\mathrm{B},+}\left(\ell_{i}\right), & 1 \leq i \leq K, j<0, \\ r_{\mathrm{B},+}\left(\ell_{i}\right) \bar{\psi}_{+}^{(\xi, j)}\left(\ell_{i} \mid Z_{+}\right), & 1 \leq i \leq K, j \geq 0, \\ 1-\bar{p}_{\mathrm{S}}^{(\xi)}\left(\ell_{i}\right), & K+1 \leq i \leq P, j<0, \\ \bar{p}_{\mathrm{S}}^{(\xi)}\left(\ell_{i}\right) \bar{\psi}_{+}^{(\xi, j)}\left(\ell_{i} \mid Z_{+}\right), & K+1 \leq i \leq P, j \geq 0,\end{cases}
$$

where $\bar{\psi}_{+}^{(\xi, j)}\left(\ell_{i} \mid Z_{+}\right)=\left\langle\bar{p}_{+}^{(\xi)}\left(\cdot, \ell_{i}\right), \psi_{+}^{(j)}\left(\cdot, \ell_{i} \mid Z_{+}\right)\right\rangle$. Then $w^{\left(I, \xi, I_{+}, \theta_{+}\right)}\left(Z_{+}\right)=\prod_{i=1}^{P} \eta_{i}\left(\gamma_{i}\right)$, if the positive 1-1 vectors $\gamma$ are equivalent representations of $\left(I_{+}, \theta_{+}\right)$. Hence generating significant GLMB children components of $(I, \xi)$ translates to generating positive 1-1 vectors with significant weights [34]. Methods for obtaining a set of positive 1-1 vectors include:

- solving a ranked assignment problem using Murty's algorithm [41], which finds the $N$ best 1-1 vectors in nonincreasing order; and

- a more efficient method using the Gibbs sampler to simulate an unordered set of significant positive 1-1 vectors [34].

\section{GLMB FILTER WITH SPAWNING}

This section presents a labeled RFS spawning model and a tractable multi-object filter for such a spawning model. In Subsection III-A we detail the labeled RFS spawning model. In Subsection III-B we derive the resulting prediction multiobject density at the next time for a given GLMB at the current time. We discuss the method of GLMB approximation which matches first moment and cardinality, then utilize it in the derivation of the multi-object filtering density at the next time in Subsection III-C. Implementation of the resulting GLMB recursion is detailed in Subsection III-D.

\section{A. Multi-object Labeled Spawning Model}

To encode ancestry information in the labels, we adhere to the following labeling convention for spawned tracks. An object spawned from a parent with label $\ell$, at time $k+1$, has label $\varsigma=(\ell, k+1, i)$, where $i$ is an index that distinguishes multiple objects simultaneously spawned by the same parent. As a result, spawn labels consist of an ancestral element, i.e., the parent's label, and a non-ancestral element that distinguishes multiple spawned objects originating simultaneously from the same parent. Hence, given the label space $\mathbb{L}_{k}$ for objects at the current time, the label space $\mathbb{T}_{k+1}$ for tracks spawned at the next time is given by $\mathbb{T}_{k+1}=\mathbb{L}_{k} \times\{k+1\} \times \mathbb{N}$.

Hereafter, in an effort to simplify notation, we revert to the convention of letting the symbol + denote the "next time" index, e.g., the label space $\mathbb{L}_{k}$ at the current time becomes $\mathbb{L}$, and the label space $\mathbb{L}_{k+1}$ at the next time becomes $\mathbb{L}_{+}$. Accordingly, we follow the same construction in [32] by letting $\mathbb{T}_{+}$denote the label space for objects spawned at the next time, then $\mathbb{L}_{+}=\mathbb{L} \cup \mathbb{T}_{+} \cup \mathbb{B}_{+}$. Note that $\mathbb{L}, \mathbb{T}_{+}$, and $\mathbb{B}_{+}$ are mutually disjoint, i.e., $\mathbb{L} \cap \mathbb{T}_{+}=\mathbb{L} \cap \mathbb{B}_{+}=\mathbb{T}_{+} \cap \mathbb{B}_{+}=\emptyset$. Hence, we can distinguish surviving, spawn, and birth objects from their labels. Fig. 1], modeled after [33, Fig.1] in the 
interest of consistency, illustrates label assignment to birth and spawn tracks.

The elements of a given current multi-object state $\mathbf{X}$ spawn new objects independently of each other. In addition, the set $\mathbf{U}$ of objects spawned at the next time by a singleobject state $\mathbf{x} \triangleq(x, \ell)$, is an $\mathrm{LMB}^{2}$ with parameter set $\left\{\left(p_{\mathrm{T}}(\mathbf{x} ; \varsigma), f_{\mathrm{T},+}(\cdot \mid \mathbf{x} ; \varsigma)\right): \varsigma \in \mathbb{T}_{+}(\mathcal{L}(\mathbf{x}))\right\}$, where

$$
\mathbb{T}_{+}(\ell) \triangleq\{(\ell, k+1)\} \times\left\{1: M_{\ell}\right\}
$$

is a finite subset set of $\mathbb{T}_{+}$. In other words, for each LMB component $\varsigma \in \mathbb{T}_{+}(\mathcal{L}(\mathbf{x}))$, the state $\mathbf{x}$ either spawns a state $\left(x_{+}, \varsigma\right)$ with probability $p_{\mathrm{T}}(\mathbf{x} ; \varsigma)$ and probability density $f_{\mathrm{T},+}\left(x_{+} \mid \mathbf{x} ; \varsigma\right)$, or it does not with probability $q_{\mathrm{T}}(\mathbf{x} ; \varsigma)=$ $1-p_{\mathrm{T}}(\mathbf{x} ; \varsigma)$. The density of the set of objects spawned from $\mathbf{x}$ can be written as

$$
\begin{aligned}
\mathbf{f}_{\mathrm{T},+}(\mathbf{U} \mid \mathbf{x})=\Delta(\mathbf{U}) 1_{\mathbb{T}_{+}(} & \mathcal{L}(\mathbf{x})) \\
& \times\left[\Phi_{\mathrm{T},+}(\mathbf{L}(\mathbf{U}))\right. \\
& \left.\left.\times{ }^{\prime} ;\right)\right]^{\mathbb{T}_{+}(\mathcal{L}(\mathbf{x}))}
\end{aligned}
$$

where

$$
\begin{array}{r}
\Phi_{\mathrm{T},+}(\mathbf{U} \mid \mathbf{x} ; \varsigma)=\sum_{\left(x_{+}, \ell_{+}\right) \in \mathbf{U}} \delta_{\varsigma}\left(\ell_{+}\right) p_{\mathrm{T}}(\mathbf{x} ; \varsigma) f_{\mathrm{T},+}\left(x_{+} \mid \mathbf{x} ; \varsigma\right) \\
+\left[1-1_{\mathcal{L}(\mathbf{U})}(\varsigma)\right] q_{\mathrm{T}}(\mathbf{x} ; \varsigma) .
\end{array}
$$

Since $\mathbf{X}$ has distinct labels, the LMB label sets $\mathbb{T}_{+}(\mathcal{L}(\mathbf{x}))$ for all $\mathbf{x} \in \mathbf{X}$ are mutually disjoint, and the set of possible labels spawned from $\mathbf{X}$ is the disjoint union

$$
\mathbb{T}_{+}(\mathcal{L}(\mathbf{X}))=\biguplus_{\mathbf{x} \in \mathbf{X}} \mathbb{T}_{+}(\mathcal{L}(\mathbf{x}))
$$

Note that when a labeled set $\mathbf{V}$ is spawned from $\mathbf{X}, \mathcal{L}(\mathbf{V}) \subseteq$ $\mathbb{T}_{+}(\mathcal{L}(\mathbf{X}))$, i.e., $1_{\mathbb{T}_{+}(\mathcal{L}(\mathbf{X}))}(\mathcal{L}(\mathbf{V}))=1$, otherwise $\mathcal{L}(\mathbf{V}) \nsubseteq$ $\mathbb{T}_{+}(\mathcal{L}(\mathbf{X}))$, i.e., $1_{\mathbb{T}_{+}(\mathcal{L}(\mathbf{X}))}(\mathcal{L}(\mathbf{V}))=0$. Hence, the inclusion $1_{\mathbb{T}_{+}(\mathcal{L}(\mathbf{X}))}(\mathcal{L}(\mathbf{V}))$ is an indicator of whether $\mathbf{V}$ is spawned by $\mathbf{X}$ or not. Moreover, if $\mathbf{V}$ is not spawned from $\mathbf{X}$, then the spawning density $\mathbf{f}_{\mathrm{T},+}(\mathbf{V} \mid \mathbf{X})=0$. On the other hand if $\mathbf{V}$ is spawned from $\mathbf{X}$, then

$$
\mathbf{V}=\biguplus_{\mathbf{x} \in \mathbf{X}} \mathbf{V} \cap\left(\mathbb{X} \times \mathbb{T}_{+}(\mathcal{L}(\mathbf{x}))\right.
$$

and since $\mathbf{V} \cap\left(\mathbb{X} \times \mathbb{T}_{+}(\mathcal{L}(\mathbf{x}))\right.$ is the set of objects spawned by $\mathbf{x}$, it follow from the Finite Set Statistics (FISST) fundamental convolution theorem [15], and arguments presented in [32] that the spawning density

$$
\mathbf{f}_{\mathrm{T},+}(\mathbf{V} \mid \mathbf{X})=\prod_{\mathbf{x} \in \mathbf{X}} \mathbf{f}_{\mathrm{T},+}\left(\mathbf{V} \cap\left(\mathbb{X} \times \mathbb{T}_{+}(\mathcal{L}(\mathbf{x})) \mid \mathbf{x}\right) .\right.
$$

Hence, $\mathbf{f}_{\mathrm{T},+}(\mathbf{V} \mid \mathbf{X})$ can be written as

$$
\begin{aligned}
\mathbf{f}_{\mathrm{T},+}(\mathbf{V} \mid \mathbf{X})=1_{\mathbb{T}_{+}(\mathcal{L}(\mathbf{X}))}(\mathcal{L}(\mathbf{V})) \\
\quad \times \prod_{\mathbf{x} \in \mathbf{X}} \mathbf{f}_{\mathrm{T},+}\left(\mathbf{V} \cap\left(\mathbb{X} \times \mathbb{T}_{+}(\mathcal{L}(\mathbf{x})) \mid \mathbf{x}\right)\right.
\end{aligned}
$$

\footnotetext{
${ }^{2}$ It is possible to derive a GLMB based spawning model, but an LMB is presented for compactness
}

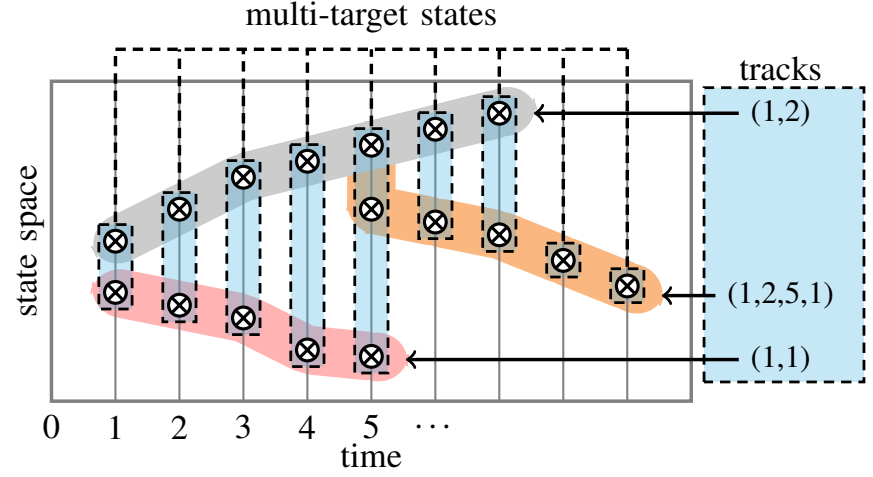

Fig. 1: An example of label assignment for birth and spawn tracks. Two tracks are born at time 1 and are assigned labels $(1,1)$ and $(1,2)$. At time 5 , a track is spawned from track $(1,2)$ and is assigned label $(1,2,5,1)$.

Substituting (21) into the above equation and noting that when $1_{\mathbb{T}_{+}(\mathcal{L}(\mathbf{X}))}(\mathcal{L}(\mathbf{V}))=1$,

$$
\begin{aligned}
1_{\mathbb{T}_{+}(\mathcal{L}(\mathbf{x}))}\left(\mathcal{L}\left(\mathbf{V} \cap\left(\mathbb{X} \times \mathbb{T}_{+}(\mathcal{L}(\mathbf{x}))\right)\right)\right. & =1, \\
\prod_{\mathbf{x} \in \mathbf{X}} \Delta\left(\mathbf{V} \cap\left(\mathbb{X} \times \mathbb{T}_{+}(\mathcal{L}(\mathbf{x}))\right)\right. & =\Delta(\mathbf{V}),
\end{aligned}
$$

we have

$$
\mathbf{f}_{\mathrm{T},+}(\mathbf{V} \mid \mathbf{X})=\Delta(\mathbf{V}) 1_{\mathbb{T}_{+}(\mathcal{L}(\mathbf{X}))}(\mathcal{L}(\mathbf{V}))\left[\Phi_{\mathrm{T},+}(\mathbf{V} \mid \cdot)\right]^{\mathbf{X}}
$$

where

$\Phi_{\mathrm{T},+}(\mathbf{V} \mid \mathbf{x})=\left[\Phi_{\mathrm{T},+}\left(\mathbf{V} \cap\left(\mathbb{X} \times \mathbb{T}_{+}(\mathcal{L}(\mathbf{x})) \mid \mathbf{x} ; \cdot\right)\right]^{\mathbb{T}_{+}(\mathcal{L}(\mathbf{x}))}\right.$

The multi-object state at the next time $\mathbf{X}_{+}=\mathbf{X}_{\mathrm{S},+} \cup \mathbf{X}_{\mathrm{T},+} \cup$ $\mathbf{X}_{\mathrm{B},+}$ is the superposition of surviving objects $\mathbf{X}_{\mathrm{S},+}=\mathbf{X}_{+} \cap$ $(\mathbb{X} \times \mathbb{L})$, birth objects $\mathbf{X}_{\mathrm{B},+}=\mathbf{X}_{+} \cap\left(\mathbb{X} \times \mathbb{B}_{+}\right)$and spawned objects $\mathbf{X}_{\mathrm{T},+}=\mathbf{X}_{+} \cap\left(\mathbb{X} \times \mathbb{T}_{+}\right)$. Since the label spaces $\mathbb{L}, \mathbb{T}_{+}$, and $\mathbb{B}_{+}$are mutually disjoint, it follows that $\mathbf{X}_{\mathrm{S},+}, \mathbf{X}_{\mathrm{T},+}$, and $\mathbf{X}_{\mathrm{B},+}$ are also mutually disjoint. Further, using the conditional independence of $\mathbf{X}_{\mathrm{S},+}, \mathbf{X}_{\mathrm{T},+}$, and $\mathbf{X}_{\mathrm{B},+}$, it follows from the FISST fundamental convolution theorem [15], [32] that the multi-object transition kernel is given by

$$
\mathbf{f}_{+}\left(\mathbf{X}_{+} \mid \mathbf{X}\right)=\mathbf{f}_{\mathrm{S},+}\left(\mathbf{X}_{\mathrm{S},+} \mid \mathbf{X}\right) \mathbf{f}_{\mathrm{T},+}\left(\mathbf{X}_{\mathrm{T},+} \mid \mathbf{X}\right) \mathbf{f}_{\mathrm{B},+}\left(\mathbf{X}_{\mathrm{B},+}\right) .
$$

\section{B. Multi-object prediction with spawning}

In general, for a multi-object transition density with spawning 26, the GLMB family is not necessarily closed under the Chapman-Kolmogorov equation

$$
\boldsymbol{\pi}\left(\mathbf{X}_{+}\right)=\int \mathbf{f}_{+}\left(\mathbf{X}_{+} \mid \mathbf{X}\right) \boldsymbol{\pi}(\mathbf{X}) \delta \mathbf{X}
$$

Proposition 1. If the current multi-object filtering density is GLMB of the form (1), then the multi-object prediction density formed by surviving, birth and spawning processes is given by

$$
\boldsymbol{\pi}\left(\mathbf{X}_{+}\right)=\Delta\left(\mathbf{X}_{+}\right) \sum_{I, \xi} w_{+}^{(I, \xi)}\left(\mathcal{L}\left(\mathbf{X}_{+}\right)\right) p^{(I, \xi)}\left(\mathbf{X}_{+}\right)
$$


where

$$
\begin{aligned}
& w_{+}^{(I, \xi)}\left(\mathcal{L}\left(\mathbf{X}_{+}\right)\right) \\
& =w^{(I, \xi)} 1_{I}\left(\mathcal{L}\left(\mathbf{X}_{\mathrm{S},+}\right)\right) 1_{\mathbb{T}_{+}(\mathcal{L}(\mathbf{X}))}\left(\mathcal{L}\left(\mathbf{X}_{\mathrm{T},+}\right)\right) 1_{\mathbb{B}_{+}}\left(\mathcal{L}\left(\mathbf{X}_{\mathrm{B},+}\right)\right) \\
& \quad \times\left[1-r_{\mathrm{B},+}\right]^{\mathbb{B}_{+}-\mathcal{L}\left(\mathbf{X}_{\mathrm{B},+}\right)}\left[r_{\mathrm{B},+}\right]^{\mathcal{L}\left(\mathbf{X}_{\mathrm{B},+}\right)}, \\
& p^{(I, \xi)}\left(\mathbf{X}_{+}\right) \\
& =\left[p_{\mathrm{B},+}\right]^{\mathbf{X}_{\mathrm{B},+}} \\
& \quad \times \prod_{\ell \in I}\left\langle\Phi_{\mathrm{S},+}\left(\mathbf{X}_{\mathrm{S},+} \mid \cdot, \ell\right) \Phi_{\mathrm{T},+}\left(\mathbf{X}_{\mathrm{T},+} \mid \cdot, \ell\right), p^{(\xi)}(\cdot, \ell)\right\rangle, \quad(30)
\end{aligned}
$$

$\mathbf{X}_{\mathrm{S},+}=\mathbf{X}_{+} \cap(\mathbb{X} \times \mathbb{L}), \mathbf{X}_{\mathrm{T},+}=\mathbf{X}_{+} \cap\left(\mathbb{X} \times \mathbb{T}_{+}\right)$, and $\mathbf{X}_{\mathrm{B},+}=$ $\mathbf{X}_{+} \cap\left(\mathbb{X} \times \mathbb{B}_{+}\right)$.

Proof. Using the Chapman-Kolmogorov equation (27) and (26) with $\mathbf{f}_{\mathrm{S},+}\left(\mathbf{X}_{\mathrm{S},+} \mid \mathbf{X}\right)$ from (5) and $\mathbf{f}_{\mathrm{T},+}\left(\mathbf{X}_{\mathrm{T},+} \mid \mathbf{X}\right)$ from (21), we have

$$
\begin{aligned}
\boldsymbol{\pi}\left(\mathbf{X}_{+}\right) & \\
= & \mathbf{f}_{\mathrm{B},+}\left(\mathbf{X}_{\mathrm{B},+}\right) \int \Delta\left(\mathbf{X}_{\mathrm{S},+}\right) 1_{\mathcal{L}(\mathbf{X})}\left(\mathcal{L}\left(\mathbf{X}_{\mathrm{S},+}\right)\right)\left[\Phi_{\mathrm{S},+}\left(\mathbf{X}_{\mathrm{S},+} \mid \cdot\right)\right]^{\mathbf{X}} \\
& \times \Delta\left(\mathbf{X}_{\mathrm{T},+}\right) 1_{\mathrm{T}_{+}(\mathcal{L}(\mathbf{X}))}\left(\mathcal{L}\left(\mathbf{X}_{\mathrm{T},+}\right)\right)\left[\Phi_{\mathrm{T},+}\left(\mathbf{X}_{\mathrm{T},+} \mid \cdot\right)\right] \\
& \times \Delta(\mathbf{X}) \sum_{I, \xi} w^{(I, \xi)} \delta_{I}(\mathcal{L}(\mathbf{X}))\left[p^{(\xi)}\right]^{\mathbf{X}} \delta \mathbf{X} \\
= & \Delta\left(\mathbf{X}_{\mathrm{S},+}\right) \Delta\left(\mathbf{X}_{\mathrm{T},+}\right) \mathbf{f}_{\mathrm{B},+}\left(\mathbf{X}_{\mathrm{B},+}\right) \sum_{I, \xi} \int \Delta(\mathbf{X}) w^{(I, \xi)} \delta_{I}(\mathcal{L}(\mathbf{X})) \\
& \times 1_{\mathcal{L}(\mathbf{X})}\left(\mathcal{L}\left(\mathbf{X}_{\mathrm{S},+}\right)\right) 1_{\mathbb{T}_{+}(\mathcal{L}(\mathbf{X}))}\left(\mathcal{L}\left(\mathbf{X}_{\mathrm{T},+}\right)\right) \\
& \times\left[\Phi_{\mathrm{S},+}\left(\mathbf{X}_{\mathrm{S},+} \mid \cdot\right) \Phi_{\mathrm{T},+}\left(\mathbf{X}_{\mathrm{T},+} \mid \cdot\right) p^{(\xi)}\right]^{\mathbf{X}} \delta \mathbf{X} \\
= & \Delta\left(\mathbf{X}_{\mathrm{S},+}\right) \Delta\left(\mathbf{X}_{\mathrm{T},+}\right) \mathbf{f}_{\mathrm{B},+}\left(\mathbf{X}_{\mathrm{B},+}\right) \sum_{I, \xi} \sum_{J \in \mathcal{F}(\mathbb{L})} w^{(I, \xi)} \delta_{I}(J) \\
& \times 1_{J}\left(\mathcal{L}\left(\mathbf{X}_{\mathrm{S},+}\right)\right) 1_{\mathbb{T}_{+}(\mathcal{L}(\mathbf{X}))}\left(\mathcal{L}\left(\mathbf{X}_{\mathrm{T},+}\right)\right) \\
& \times \prod_{\ell \in I}\left\langle\Phi_{\mathrm{S},+}\left(\mathbf{X}_{\mathrm{S},+} \mid \cdot, \ell\right) \Phi_{\mathrm{T},+}\left(\mathbf{X}_{\mathrm{T},+} \mid \cdot, \ell\right), p^{(\xi)}(\cdot, \ell)\right\rangle(33)
\end{aligned}
$$

where the last line follows from [32, Lemma 3]. Using $\mathbf{f}_{\mathrm{B},+}\left(\mathbf{X}_{\mathrm{B},+}\right)$ from [6], $\Delta\left(\mathbf{X}_{+}\right)=$ $\Delta\left(\mathbf{X}_{\mathrm{S},+}\right) \Delta\left(\mathbf{X}_{\mathrm{T},+}\right) \Delta\left(\mathbf{X}_{\mathrm{B},+}\right)$, and noting that the only non-zero inner summand occurs when $I=J$, we have 28.

Implicit in (28) is that, even though the survival and spawn RFSs are mutually disjoint due to their labels (see (26)), they are both conditioned on the same multi-object state X. Further, the objects spawned by a state $\mathbf{x} \in \mathbf{X}$ and its state at the next time (if survived) are all distinct, but conditioned on $\mathbf{x}$.

\section{Multi-Object Update with Spawning}

Since the predicted multi-object density is not a GLMB, the updated multi-object density

$$
\boldsymbol{\pi}_{+}\left(\mathbf{X}_{+} \mid Z_{+}\right)=\frac{\boldsymbol{\pi}\left(\mathbf{X}_{+}\right) g\left(Z_{+} \mid \mathbf{X}_{+}\right)}{\int \boldsymbol{\pi}(\mathbf{X}) g\left(Z_{+} \mid \mathbf{X}\right) \delta \mathbf{X}} .
$$

with the standard multi-object likelihood, is also not a GLMB. One strategy of using the GLMB filter to track with spawnings is to approximate the multi-object prediction density (28) by a GLMB prior to performing a measurement update. In this work we propose a more prudent approach whereby the GLMB approximation is performed on the updated multiobject density to reduce information loss, albeit at the cost of increased complexity. Such approximation can be achieved by finding a GLMB that matches the multi-object filtering density in the first moment and cardinality, and we do so as follows.

An arbitrary labeled multi-object density on $\mathcal{F}(\mathbb{X} \times \mathbb{L})$ can be writen in the form [36. Proposition 3],

$$
\boldsymbol{\pi}(\mathbf{X})=\Delta(\mathbf{X}) \sum_{c \in \mathbb{C}} w^{(c)}(\mathcal{L}(\mathbf{X})) p^{(c)}(\mathbf{X})
$$

where $\mathbb{C}$ is a discrete index set, the weights $w^{(c)}(\cdot)$ satisfy (2), and with $n=|\mathbf{X}|$,

$$
\int p^{(c)}\left(\left\{\left(x_{1}, \ell_{1}\right), \ldots,\left(x_{n}, \ell_{n}\right)\right\}\right) d\left(x_{1}, \ldots, x_{n}\right)=1 .
$$

Moreover, it was shown in [36, Proposition 3], that such a labeled multi-object density can be approximated by the GLMB

$$
\hat{\boldsymbol{\pi}}(\mathbf{X})=\Delta(\mathbf{X}) \sum_{(c, I) \in \mathbb{C} \times \mathcal{F}(\mathbb{L})} \delta_{I}(\mathcal{L}(\mathbf{X})) \hat{w}^{(c, I)}\left[\hat{p}^{(c, I)}\right]^{\mathbf{X}}
$$

where

$$
\begin{aligned}
& \hat{w}^{(c, I)}=w^{(c)}(I), \\
& \hat{p}^{(c, I)}(x, \ell)=1_{I}(\ell) p_{I-\{\ell\}}^{(c)}(x, \ell), \\
& p_{\left\{\ell_{1}, \ldots, \ell_{n}\right\}}^{(c)}(x, \ell)= \\
& \int p^{(c)}\left(\left\{(x, \ell),\left(x_{1}, \ell_{1}\right), \ldots,\left(x_{n}, \ell_{n}\right)\right\}\right) d\left(x_{1}, \ldots, x_{n}\right) .
\end{aligned}
$$

A salient feature of this approximation method is that both the cardinality distribution and PHD of $\pi$ are preserved. Additionally, note that $\mathbb{C}$ can take the form of any discrete index set, including the set of indices for the Cartesian product of a collection of finites subsets of some label space and an association history space, i.e., letting $\mathbb{C}=\mathcal{F}(\mathbb{L}) \times \Xi$ is possible.

The exact form of the multi-object filtering density at the next time, and its GLMB approximation as per the result above, is given below in Proposition 2.

Proposition 2. If the current filtering density is GLMB of form (1) and given the multi-object likelihood (8), then the multi-object filtering density at the next time is given by

$$
\begin{aligned}
& \boldsymbol{\pi}_{+}\left(\mathbf{X}_{+} \mid Z_{+}\right) \\
& \propto \Delta\left(\mathbf{X}_{+}\right) \sum_{I, \xi, \theta_{+}} w_{+}^{(I, \xi)}\left(\mathcal{L}\left(\mathbf{X}_{+}\right)\right)\left[p_{\mathrm{B},+} \psi_{+}^{\left(\theta_{+}\right)}\left(\cdot \mid Z_{+}\right)\right]^{\mathbf{X}_{\mathrm{B},+}} \\
& \quad \times p_{+}^{\left(I, \xi, \theta_{+}\right)}\left(\mathbf{X}_{\mathrm{S},+} \cup \mathbf{X}_{\mathrm{T},+} \mid Z_{+}\right)
\end{aligned}
$$

where $I \in \mathcal{F}(\mathbb{L}), \xi \in \Xi, \theta_{+} \in \Theta_{+}\left(\mathcal{L}\left(\mathbf{X}_{+}\right)\right), \mathbf{X}_{\mathrm{B},+}=\mathbf{X}_{+} \cap$ $\left(\mathbb{X} \times \mathbb{B}_{+}\right), \mathbf{X}_{\mathrm{S},+}=\mathbf{X}_{+} \cap(\mathbb{X} \times \mathbb{L}), \mathbf{X}_{\mathrm{T},+}=\mathbf{X}_{+} \cap\left(\mathbb{X} \times \mathbb{T}_{+}\right)$, and

$$
\begin{aligned}
& p_{+}^{\left(I, \xi, \theta_{+}\right)}\left(\mathbf{X}_{\mathrm{S},+} \cup \mathbf{X}_{\mathrm{T},+} \mid Z_{+}\right) \\
& =\left[\psi_{+}^{\left(\theta_{+}\right)}\left(\cdot \mid Z_{+}\right)\right]^{\mathbf{X}_{\mathrm{S},+} \cup \mathbf{X}_{\mathrm{T},+}} \\
& \quad \times \prod_{\ell \in I}\left\langle\Phi_{\mathrm{S},+}\left(\mathbf{X}_{\mathrm{S},+} \mid \cdot, \ell\right) \Phi_{\mathrm{T},+}\left(\mathbf{X}_{\mathrm{T},+} \mid \cdot, \ell\right), p^{(\xi)}(\cdot, \ell)\right\rangle .
\end{aligned}
$$




$$
\begin{aligned}
& \hat{\boldsymbol{\pi}}_{+}\left(\mathbf{X}_{+} \mid Z_{+}\right)=\Delta\left(\mathbf{X}_{+}\right) \sum_{I, \xi, I_{+}, \theta_{+}} \delta_{I_{+}}\left(\mathcal{L}\left(\mathbf{X}_{+}\right)\right) \hat{w}_{+}^{\left(I, \xi, I_{+}, \theta_{+}\right)}\left(Z_{+}\right)\left[p_{\mathrm{B},+} \psi_{+}^{\left(\theta_{+}\right)}\left(\cdot \mid Z_{+}\right)\right]^{\mathbf{X}_{\mathrm{B},+}} \frac{\left[\hat{p}_{+}^{\left(I, \xi, I_{+}, \theta_{+}\right)}\left(\cdot \mid Z_{+}\right)\right]^{\mathbf{X}_{\mathrm{S},+} \cup \mathbf{X}_{\mathrm{T},+}}}{\left[\bar{p}_{+}^{\left(I, \xi, I_{+}, \theta_{+}\right)}\left(\cdot \mid Z_{+}\right)\right]^{I_{+}}} \\
& \hat{w}_{+}^{\left(I, \xi, I_{+}, \theta_{+}\right)}\left(Z_{+}\right)=\frac{w_{+}^{(I, \xi)}\left(I_{+}\right)\left[\bar{p}_{+}^{\left(I, \xi, I_{+}, \theta_{+}\right)}\left(\cdot \mid Z_{+}\right)\right]^{I_{+}}}{\sum_{I, \xi, I_{+}, \theta_{+}} w_{+}^{(I, \xi)}\left(I_{+}\right)\left[\bar{p}_{+}^{\left(I, \xi, I_{+}, \theta_{+}\right)}\left(\cdot \mid Z_{+}\right)\right]^{I_{+}}} \\
& \hat{p}_{+}^{\left(I, \xi, I_{+}, \theta_{+}\right)}\left(x_{+}, \ell_{+} \mid Z_{+}\right)=1_{I_{+}}\left(\ell_{+}\right) \int p_{+}^{\left(I, \xi, \theta_{+}\right)}\left(\left\{\left(x_{+}, \ell_{+}\right),\left(x_{1,+}, \ell_{1,+}\right), \ldots,\left(x_{n,+}, \ell_{n,+}\right)\right\} \mid Z_{+}\right) d\left(x_{1,+}, \ldots, x_{n,+}\right) \\
& \bar{p}_{+}^{\left(I, \xi, I_{+}, \theta_{+}\right)}\left(\ell_{+} \mid Z_{+}\right)=1_{\mathbb{B}_{+}}\left(\ell_{+}\right)\left\langle p_{\mathrm{B},+}\left(\cdot, \ell_{+}\right), \psi_{+}^{\left(\theta_{+}\right)}\left(\cdot \mid Z_{+}\right)\right\rangle+\left(1-1_{\mathbb{B}_{+}}\left(\ell_{+}\right)\right)\left\langle\hat{p}_{+}^{\left(I, \xi, I_{+}, \theta_{+}\right)}\left(\cdot, \ell_{+} \mid Z_{+}\right), 1\right\rangle
\end{aligned}
$$

Moreover, it can be approximated by the GLMB given by (42)-(45) [see top of page] which preserves the first moment and cardinality distribution, where $I_{+} \in \mathbb{L}_{+}$.

Proof. With $g\left(Z_{+} \mid \mathbf{X}_{+}\right)$from (8) and $\boldsymbol{\pi}\left(\mathbf{X}_{+}\right)$from (28), we have

$$
\begin{aligned}
\boldsymbol{\pi}_{+} & \left(\mathbf{X}_{+} \mid Z_{+}\right) \\
\propto & \boldsymbol{\pi}\left(\mathbf{X}_{+}\right) g\left(Z_{+} \mid \mathbf{X}_{+}\right), \\
= & \Delta\left(\mathbf{X}_{+}\right) \sum_{I, \xi, \theta_{+}} w_{+}^{(I, \xi)}\left(\mathcal{L}\left(\mathbf{X}_{+}\right)\right) p_{+}^{(I, \xi)}\left(\mathbf{X}_{+}\right) \\
& \times\left[\psi_{+}^{\left(\theta_{+}\right)}\left(\cdot \mid Z_{+}\right)\right]^{\mathbf{X}_{+}}, \\
= & \Delta\left(\mathbf{X}_{+}\right) \sum_{I, \xi, \theta_{+}} w_{+}^{(I, \xi)}\left(\mathcal{L}\left(\mathbf{X}_{+}\right)\right)\left[p_{\mathrm{B},+} \psi_{+}^{\left(\theta_{+}\right)}\left(\cdot \mid Z_{+}\right)\right]^{\mathbf{X}_{\mathrm{B},+}} \\
& \times \prod_{\ell \in I}\left\langle\Phi_{\mathrm{S},+}\left(\mathbf{X}_{\mathrm{S},+} \mid \cdot, \ell\right) \Phi_{\mathrm{T},+}\left(\mathbf{X}_{\mathrm{T},+} \mid \cdot, \ell\right), p^{(\xi)}(\cdot, \ell)\right\rangle \\
& \times\left[\psi_{+}^{(\theta)}\left(\cdot \mid Z_{+}\right)\right]^{\mathbf{X}_{\mathrm{S},+} \cup \mathbf{X}_{\mathrm{T},+}} \\
= & \Delta\left(\mathbf{X}_{+}\right) \sum_{I, \xi, \theta_{+}} w_{+}^{(I, \xi)}\left(\mathcal{L}\left(\mathbf{X}_{+}\right)\right)\left[p_{\mathrm{B},+} \psi_{+}^{(\theta)}\left(\cdot \mid Z_{+}\right)\right]^{\mathbf{X}_{\mathrm{B},+}} \\
& \times p_{+}^{\left(I, \xi, \theta_{+}\right)}\left(\mathbf{X}_{\mathrm{S},+} \cup \mathbf{X}_{\mathrm{T},+} \mid Z_{+}\right) .
\end{aligned}
$$

We now apply the GLMB approximation (36) to (49), and note that determining the product of the marginals of the single-object birth densities encapsulated in $\left[p_{\mathrm{B},+} \psi_{+}^{\left(\theta_{+}\right)}\left(\cdot \mid Z_{+}\right)\right]^{\mathbf{X}_{\mathrm{B},+}}$ is redundant. Only the single-object densities encapsulated in $p_{+}^{\left(I, \xi, \theta_{+}\right)}\left(\mathbf{X}_{\mathrm{S},+} \cup \mathbf{X}_{\mathrm{T},+} \mid Z_{+}\right)$require marginalization. Hence, applying the GLMB approximation from 36 gives

$$
\begin{aligned}
\hat{\boldsymbol{\pi}}_{+}\left(\mathbf{X}_{+} \mid Z_{+}\right)=\hat{C} & \Delta\left(\mathbf{X}_{+}\right) \sum_{I, \xi, I_{+}, \theta_{+}} \delta_{I_{+}}\left(\mathcal{L}\left(\mathbf{X}_{+}\right)\right) \\
& \times w_{+}^{(I, \xi)}\left(\mathcal{L}\left(\mathbf{X}_{+}\right)\right)\left[p_{\mathrm{B},+} \psi_{+}^{(\theta)}\left(\cdot \mid Z_{+}\right)\right]^{\mathbf{X}_{\mathrm{B},+}} \\
& \times\left[\hat{p}^{\left(I, \xi, I_{+}, \theta_{+}\right)}\left(\cdot \mid Z_{+}\right)\right]^{\mathbf{X}_{\mathrm{S},+} \cup \mathbf{X}_{\mathrm{T},+}}
\end{aligned}
$$

where $I_{+} \in \mathcal{F}\left(\mathbb{L}_{+}\right), \hat{p}^{\left(I, \xi, I_{+}, \theta_{+}\right)}\left(\cdot \mid Z_{+}\right)$is defined in (44), and

$$
\begin{aligned}
\hat{C}^{-1}= & \sum_{I, \xi, I_{+}, \theta_{+}} \int \Delta\left(\mathbf{X}_{+}\right) \delta_{I_{+}}\left(\mathcal{L}\left(\mathbf{X}_{+}\right)\right) w^{(I, \xi)}\left(\mathcal{L}\left(\mathbf{X}_{+}\right)\right) \\
& \times\left[p_{\mathrm{B},+} \psi_{+}^{\left(\theta_{+}\right)}\left(\cdot \mid Z_{+}\right)\right]^{\mathbf{X}_{\mathrm{B},+}} \\
& \times\left[\hat{p}_{+}^{\left(I, \xi, I_{+}, \theta_{+}\right)}\left(\cdot \mid Z_{+}\right)\right]^{\mathbf{X}_{\mathrm{S},+} \cup \mathbf{X}_{\mathrm{T},+}} \delta \mathbf{X}_{+} \\
= & \sum_{I, \xi, I_{+}, \theta_{+}} \sum_{L \subseteq \mathbb{L}_{+}} \delta_{I_{+}}(L) w_{+}^{(I, \xi)}(L) \\
& \times \prod_{\ell_{+} \in L} \bar{p}_{+}^{\left(I, \xi, I_{+}, \theta_{+}\right)}\left(\ell_{+} \mid Z_{+}\right) \\
= & \sum_{I, \xi, I_{+}, \theta_{+}} w_{+}^{(I, \xi)}\left(I_{+}\right)\left[\bar{p}_{+}^{\left(I, \xi, I_{+}, \theta_{+}\right)}\left(\cdot \mid Z_{+}\right)\right]^{I_{+}}
\end{aligned}
$$

where (52) follows from [32, Lemma 3], which simplifies in (53) since the only non-zero inner summand occurs when $L=$ $I_{+}$, and $\bar{p}_{+}^{\left(I, \xi, I_{+}, \theta_{+}\right)}\left(\ell_{+} \mid Z_{+}\right)$is defined in 45. Substituting (53) into (50) we have (42) [see top of page].

\section{Efficient Implementation}

Expanding on the material discussed in Section II-D in this section we leverage the joint prediction and update of the fast GLMB implementation to truncate the number of components used to generate the updated multi-object density in (42). Note that a GLMB density of form (1) at the current time can be represented by

$$
\left\{\left(I^{(h)}, \xi^{(h)}, w^{(h)}, p^{(h)}\right)\right\}_{h=1}^{H},
$$

which is an enumeration of the set of density parameters $\left\{\left(w^{(I, \xi)}, p^{(\xi)}\right):(I, \xi) \in \mathcal{F}(\mathbb{L}) \times \Xi\right\}$ where $\left.w^{(h)} \triangleq w^{\left(I^{(h)}, \xi^{(h)}\right.}\right)$ and $p^{(h)} \triangleq p^{\left(\xi^{(h)}\right)}$. The objective is to generate a parameter set

$$
\left\{\left(I_{+}^{\left(h_{+}\right)}, \xi_{+}^{\left(h_{+}\right)}, w_{+}^{\left(h_{+}\right)}, p_{+}^{\left(h_{+}\right)}\right)\right\}_{h_{+}=1}^{H_{+}}
$$

that represents the GLMB density at the next time given by (42). Following the development in [34, Section III-E], the first step toward doing so requires drawing $H_{+}^{\max }$ samples from the distribution $\pi$ given as

$$
\pi(I, \xi) \propto w^{(I, \xi)}
$$


noting that $T_{+}^{(h)}$ duplicates of a distinct sample $\left(I^{(h)}, \xi^{(h)}\right)$ can be drawn. Then, we determine a set of $\tilde{T}_{+}^{(h)}$ candidate components of the form

$$
\left\{\left(I_{+}^{(h, t)}, \theta_{+}^{(h, t)}\right)\right\}_{t=1}^{\tilde{T}_{+}^{(h)}}
$$

from each (distinct) $\left(I^{(h)}, \xi^{(h)}\right)$ that together yield significant weights, as in $\hat{w}_{+}^{\left(I, \xi, I_{+}, \theta_{+}\right)}\left(Z_{+}\right)$in (42).

Recall from Section II-D that a set of positive 1-1 vectors $\gamma$ specifies a significant weight $\hat{w}_{+}^{\left(I, \xi, I_{+}, \theta_{+}\right)}\left(Z_{+}\right)$, if $\gamma$ generates a significant $\omega^{(\gamma)}=\prod_{i=1}^{P} \eta_{i}\left(\gamma_{i}\right)$. Hence, determining a set of candidate components amounts to finding sets of $\gamma$ 's that yield $\omega^{(\gamma)}$ above a given threshold; it follows that such vector sets can be generated using 42. However, complexity of the GLMB filter is naturally increased with the inclusion of spawn modeling and the involvement of marginalization in the truncation procedure is inefficient, especially in cases where many GLMB components are ultimately discarded. Therefore, we exploit the following proposal density for the purpose of generating candidate components (57) in an effort to offset complexity and minimize inefficiency.

Definition 1. Given a GLMB density (1) at the current time, let the proposal density $\tilde{\pi}$ at the next time be of form (10) such that

$$
\begin{aligned}
\tilde{\boldsymbol{\pi}}_{+}\left(\mathbf{X}_{+} \mid Z_{+}\right) \propto \Delta & \left(\mathbf{X}_{+}\right) \sum_{h=1}^{H} \sum_{t=1}^{\tilde{T}_{+}^{(h)}} w^{(h)} \tilde{w}_{+}^{(h, t)}\left(Z_{+}\right) \\
& \times \delta_{I_{+}^{(h)}}\left(\mathcal{L}\left(\mathbf{X}_{+}\right)\right)\left[\tilde{p}^{(h, t)}\left(Z_{+}\right)\right]^{\mathbf{X}_{+}}
\end{aligned}
$$

where $\psi_{+}^{(h, t)} \triangleq \psi_{+}^{\left(\theta_{+}^{(h, t)}\right)}, \tilde{\psi}_{+}^{(h, t)} \triangleq \tilde{\psi}_{+}^{\left(\theta_{+}^{(h, t)}\right)}$,

$$
\begin{aligned}
& \tilde{w}_{+}^{(h, t)}\left(Z_{+}\right)=\left[r_{\mathrm{B},+}\right]^{\mathbb{B}_{+} \cap I_{+}^{(h, t)}}\left[1-r_{\mathrm{B},+}\right]^{\mathbb{B}_{+}-I_{+}^{(h, t)}} \\
& \times\left[\bar{p}_{\mathrm{S}}^{(h)}\right]^{I^{(h)} \cap I_{+}^{(h, t)}}\left[1-\bar{p}_{\mathrm{S}}^{(h)}\right]^{I^{(h)}-I_{+}^{(h, t)}} \\
& \times\left[\bar{p}_{\mathrm{T}}^{(h)}\right]^{\mathbb{T}_{+} \cap I_{+}^{(h, t)}}\left[1-\bar{p}_{\mathrm{T}}^{(h)}\right]^{\mathbb{T}_{+}-I_{+}^{(h, t)}} \\
& \times\left[\tilde{\psi}_{+}^{(h, t)}\left(\cdot \mid Z_{+}\right)\right]^{I_{+}^{(h, t)}}, \\
& \tilde{p}^{(h, t)}\left(x_{+}, \ell_{+} \mid Z_{+}\right)=\frac{\tilde{p}_{+}^{(h)}\left(x_{+}, \ell_{+}\right) \psi^{(h, t)}\left(x_{+}, \ell_{+} \mid Z_{+}\right)}{\tilde{\psi}^{(h, t)}\left(x_{+}, \ell_{+} \mid Z_{+}\right)}, \\
& \tilde{p}_{+}^{(h)}\left(x_{+}, \ell_{+}\right)=1_{\mathbb{B}_{+}}\left(\ell_{+}\right) p_{\mathrm{B},+}\left(x_{+}, \ell_{+}\right) \\
& +1_{\mathbb{L}}\left(\ell_{+}\right) \tilde{p}_{\mathrm{S}}^{(h)}\left(x_{+}, \ell_{+}\right) \\
& +1_{\mathbb{T}_{+}}\left(\ell_{+}\right) \tilde{p}_{\mathrm{T}}^{(h)}\left(x_{+}, \ell_{+}\right), \\
& \tilde{p}_{\mathrm{S}}^{(h)}\left(x_{+}, \ell_{+}\right)=\frac{\left\langle p_{\mathrm{S}}\left(\cdot, \ell_{+}\right) f_{\mathrm{S},+}\left(x_{+} \mid \cdot, \ell\right), p^{(h)}\left(\cdot, \ell_{+}\right)\right\rangle}{\bar{p}_{\mathrm{S}}^{(h)}\left(\ell_{+}\right)}, \\
& \tilde{p}_{\mathrm{T}}^{(h)}\left(x_{+}, \ell_{+}\right)=\frac{\left\langle p_{\mathrm{T}}\left(\ell_{+}\right) f_{\mathrm{T},+}\left(x_{+} \mid \cdot, \ell\right), p^{(h)}(\cdot, \ell)\right\rangle}{\bar{p}_{\mathrm{T}}^{(h)}\left(\ell_{+}\right)}, \\
& \bar{p}_{\mathrm{S}}^{(h)}\left(\ell_{+}\right)=\left\langle p^{(h)}(\cdot, \ell), p_{\mathrm{S}}\left(\ell_{+}\right)\right\rangle, \\
& \bar{p}_{\mathrm{T}}^{(h)}\left(\ell_{+}\right)=\left\langle p^{(h)}(\cdot, \ell), p_{\mathrm{T}}\left(\ell_{+}\right)\right\rangle, \\
& \tilde{\psi}^{(h, t)}\left(x_{+}, \ell_{+} \mid Z_{+}\right)=\left\langle\tilde{p}_{+}^{(h)}\left(x_{+}, \ell_{+}\right), \psi^{(h, t)}\left(x_{+}, \ell_{+} \mid Z_{+}\right)\right\rangle \text {. }
\end{aligned}
$$

We enumerate $Z_{+}=\left\{z_{1:\left|Z_{+}\right|}\right\}, \mathbb{B}_{+}=\left\{\ell_{1: K}\right\}, I^{(h)}=$ $\left\{\ell_{K+1: L}\right\}$, along with the additional set of spawn labels at the next time $\mathbb{T}_{+}\left(I^{(h)}\right)=\left\{\ell_{L+1: P}\right\}$. Next we define a $P$ dimensional vector $\gamma^{(h, t)}$ that inherits the 1-1 mapping from $\theta_{+}^{(h, t)}$ (see (17)), then we recover $\left(I_{+}^{(h, t)}, \theta_{+}^{(h, t)}\right)$ via

$$
\begin{aligned}
I_{+}^{(h, t)} & =\left\{\ell_{i} \in \mathbb{B}_{+} \cup I^{(h)} \cup \mathbb{T}_{+}\left(I^{(h)}\right): \gamma_{i}^{(h, t)} \geq 0\right\}, \\
\theta_{+}^{(h, t)}\left(\ell_{i}\right) & =\gamma_{i}^{(h, t)},
\end{aligned}
$$

such that $\theta_{+}^{(h, t)}: I_{+}^{(h, t)} \rightarrow\left\{0:\left|Z_{+}\right|\right\}$. Then, for each $j \in$ $\left\{-1:\left|Z_{+}\right|\right\}$define

$$
\eta_{i}^{(h)}(j)= \begin{cases}1-r_{\mathrm{B},+}\left(\ell_{i}\right), & \ell_{i} \in \mathbb{B}_{+}, j<0, \\ r_{\mathrm{B},+}\left(\ell_{i}\right) \tilde{\psi}_{+}^{(h, j)}\left(\ell_{i} \mid Z_{+}\right), & \ell_{i} \in \mathbb{B}_{+}, j \geq 0, \\ 1-\bar{p}_{\mathrm{S}}^{(h)}\left(\ell_{i}\right), & \ell_{i} \in I^{(h)}, j<0, \\ \bar{p}_{\mathrm{S}}^{(h)}\left(\ell_{i}\right) \tilde{\psi}_{+}^{(h, j)}\left(\ell_{i} \mid Z_{+}\right), & \ell_{i} \in I^{(h)}, j \geq 0, \\ 1-\bar{p}_{\mathrm{T}}^{(h)}\left(\ell_{i}\right), & \ell_{i} \in \mathbb{T}_{+}\left(I^{(h)}\right), j<0, \\ \bar{p}_{\mathrm{T}}^{(h)}\left(\ell_{i}\right) \tilde{\psi}_{+}^{(h, j)}\left(\ell_{i} \mid Z_{+}\right), & \ell_{i} \in \mathbb{T}_{+}\left(I^{(h)}\right), j \geq 0,\end{cases}
$$

assuming that, for all $i \in\{1: P\}, \bar{p}_{\mathrm{S}}^{(h)}\left(\ell_{i}\right) \in(0,1), \bar{p}_{\mathrm{T}}^{(h)}\left(\ell_{i}\right) \in$ $(0,1)$, and $\bar{p}_{\mathrm{D},+}^{(h)}\left(\ell_{i}\right) \triangleq\left\langle\tilde{p}_{+}^{(h)}\left(\cdot, \ell_{i}\right), p_{\mathrm{D},+}\left(\cdot, \ell_{i}\right)\right\rangle \in(0,1)$. We use (68) in conjunction with the Gibbs sampler to yield mostly high-weighted positive 1-1 vectors $\gamma^{(h, t)}$, then we convert each $\gamma^{(h, t)}$ to candidate component $\left(I_{+}^{(h, t)}, \theta_{+}^{(h, t)}\right)$ using 67).

Moving forward, the candidate components we determine using the proposal density $(58)-(68)$ are subsequently used to generate the GLMB density in (42). For each sample component $\left(I^{(h)}, \xi^{(h)}\right)$ and each of its candidate components 57. formed using (67) and (68), we generate intermediate components of form $\left(I_{+}^{(h, t)}, \xi_{+}^{(h, t)}, w_{+}^{(h, t)}, p_{+}^{(h, t)}\right)$. Letting

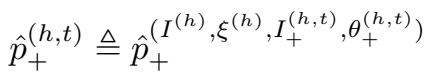

and

$$
\bar{p}_{+}^{(h, t)} \triangleq \bar{p}_{+}^{\left(I^{(h)}, \xi^{(h)}, I_{+}^{(h, t)}, \theta_{+}^{(h, t)}\right)},
$$

we compute

$$
\begin{aligned}
\bar{w}_{+}^{(h, t)}= & w^{(h)}\left[r_{\mathrm{B},+}\right]^{\mathbb{B}_{+} \cap I_{+}^{(h, t)}}\left[1-r_{\mathrm{B},+}\right]^{\mathbb{B}_{+}-I_{+}^{(h, t)}} \\
& \times\left[\bar{p}_{+}^{(h, t)}\left(\cdot \mid Z_{+}\right)\right]^{I_{+}^{(h, t)}}, \\
p_{+}^{(h, t)}\left(\cdot, \ell_{i}\right) \propto & 1_{\mathbb{B}_{+}}\left(\ell_{i}\right) p_{\mathrm{B},+}\left(\cdot, \ell_{i}\right) \psi_{+}^{(h, t)}\left(\cdot, \ell_{i} \mid Z_{+}\right) \\
& +\left(1-1_{\mathbb{B}_{+}}\left(\ell_{i}\right)\right) \hat{p}_{+}^{(h, t)}\left(\cdot, \ell_{i} \mid Z_{+}\right), \\
\text {define } \xi_{+}^{(h, t)}= & \left(\xi^{(h)}, \theta_{+}^{(h, t)}\right), \text { and let } \\
& \hat{C}=\sum_{h, t} \bar{w}_{+}^{(h, t)}\left[\bar{p}_{+}^{(h, t)}\left(\cdot \mid Z_{+}\right)\right]^{I_{+}^{(h, t)}}
\end{aligned}
$$

be a normalizing constant. Equations 69-(71) follow directly from (42)-45. Algorithm 1 (see appendix) summarizes the joint prediction and update procedure for one iteration, including how the intermediate component set $\left\{\left(I_{+}^{(h, t)}, \xi_{+}^{(h, t)}, w_{+}^{(h, t)}, p_{+}^{(h, t)}\right)\right\}_{h, t=1,1}^{H, \tilde{T}_{+}^{(h)}}$ is formed.

Note that a given set $\left\{\left(I_{+}^{(h, t)}, \xi_{+}^{(h, t)}, p_{+}^{(h, t)}\right)\right\}_{h, t=1,1}^{H, \tilde{T}_{+}^{(h)}}$ may not be unique, accordingly, the parameter set (55) is determined by summing all $w_{+}^{(h, t)}$ with $\left(I_{+}^{(h, t)}, \xi_{+}^{(h, t)}\right)=\left(I_{+}^{\left(h_{+}\right)}, \xi_{+}^{\left(h_{+}\right)}\right)$, 


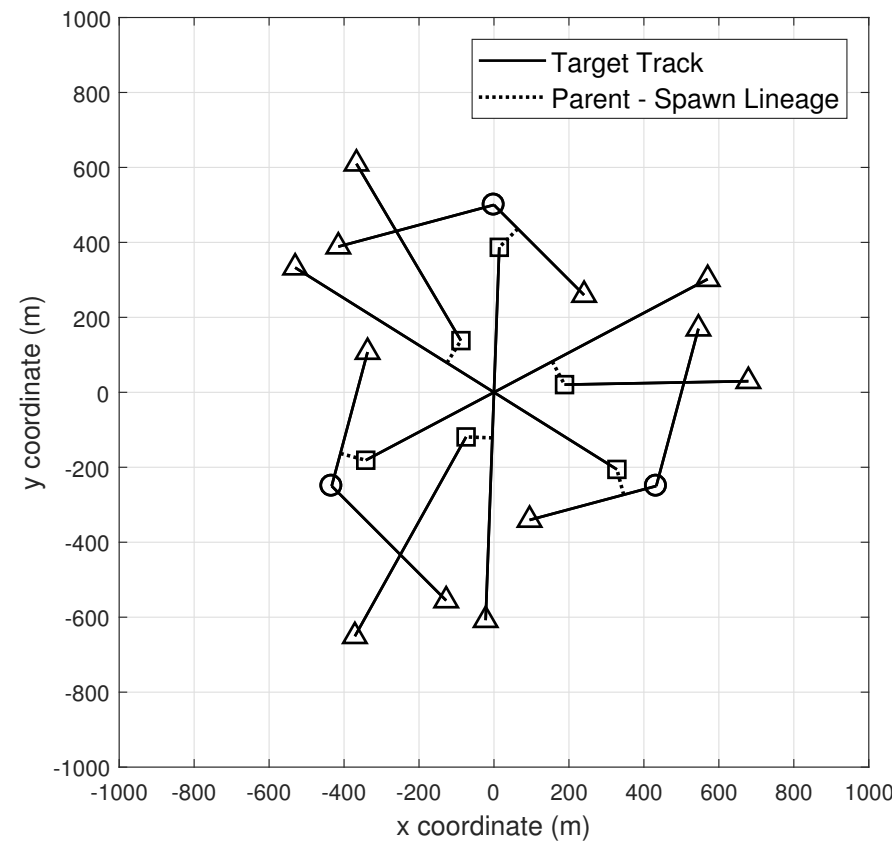

Fig. 2: Object trajectories in the $x y$ plane. A circle " $\bigcirc$ " indicates where an object is born, a square " $\square$ " indicates where a spawned object may be detected, and a triangle " $\triangle$ " indicates where an object dies.

then normalizing the weights $w_{+}^{(h, t)}$. This procedure follows from the relationship presented in 16 and is summarized in Algorithm 2, which comes from the bottom portion of [34. Algorithm 2] and is replicated here for convenience. All algorithms are relegated to the Appendix and follow the format of those presented in [34] in the interest of consistency. Additionally, we use the Gibbs and Unique functions as described in [34, Algorithm 1] and [34, Section III-E], respectively.

\section{Simulation}

A linear Gaussian example is used to verify the proposed GLMB filter and compare its performance to the CPHD filter; both filters incorporate object spawning. Fig. 2 illustrates the multiple trajectories in a $[-1000,1000] \mathrm{m} \times[-1000,1000] \mathrm{m}$ surveillance region considered in this scenario. Over the $100 \mathrm{~s}$ scenario duration, the number of objects varies due to birth, spawning, and death. In total, there are 6 spontaneous births and 6 spawning events.

At the start, an object is born in each of the three birth regions. Each birth object goes on to generate a single first generation spawn, then dies. After crossing at the origin at time $k=45$, each spawn object generates a single second generation spawn. Towards the end, an object appears in each birth region that goes on to cross paths with a second generation spawn; crossings occur at times $k=82, k=$ 84 , and $k=86$ at positions $(-250,-433),(-260,430)$, and $(507,26)$, respectively.

The single-object state describing an object's planar position and velocity coordinates is $x_{+}=\left[p_{x,+}, p_{y,+}, \dot{p}_{x,+}, \dot{p}_{y,+}\right]^{T}$. Each object has a probability of survival $p_{\mathrm{S}}=0.99$ and follows linear Gaussian dynamics with transition density $f_{\mathrm{S},+}\left(x_{+}, \ell_{+} \mid x, \ell\right)=\mathcal{N}\left(x_{+} ; F x, Q\right)$ such that

$$
F=\left[\begin{array}{cc}
I_{2} & \Delta I_{2} \\
0_{2} & I_{2}
\end{array}\right], \quad Q=\sigma_{\nu}^{2}\left[\begin{array}{cc}
\frac{\Delta^{4}}{4} I_{2} & \frac{\Delta^{3}}{2} I_{2} \\
\frac{\Delta^{3}}{2} I_{2} & \Delta^{2} I_{2}
\end{array}\right],
$$

where $\Delta=1 \mathrm{~s}, \sigma_{\nu}=1 \mathrm{~m} \mathrm{~s}^{-2}$, and $I_{n}$ and $0_{n}$ denote the $n \times n$ identity and zero matrices, respectively.

Each object is detected with probability $p_{\mathrm{D},+}=0.88$ and each object generated measurement $z_{+}=\left[z_{x,+}, z_{y,+}\right]^{T}$ consists of the object's position with noise added to each component. Measurements follow the linear Gaussian measurement model $g_{+}\left(z_{+} \mid x_{+}, \ell_{+}\right)=\mathcal{N}\left(z_{+} ; H x_{+}, R\right)$ such that

$$
H=\left[\begin{array}{ll}
I_{2} & 0_{2}
\end{array}\right], \quad R=\sigma_{\epsilon}^{2} I_{2},
$$

where $\sigma_{\epsilon}=10 \mathrm{~m}$. Clutter is modeled as a Poisson RFS with an average intensity of $\lambda_{c}=1.65 \times 10^{-5} \mathrm{~m}^{-2}$ yielding an average of 66 clutter returns per scan.

Objects can appear either by birth or spawning. The birth model is an LMB RFS with parameters $\pi_{\mathrm{B},+}=$ $\left\{r_{\mathrm{B},+}\left(\ell_{i}\right), p_{\mathrm{B},+}\left(\ell_{i}\right)\right\}_{i=1}^{3} \quad$ where $r_{\mathrm{B},+}\left(\ell_{i}\right)=0.02$ and $p_{\mathrm{B},+}\left(\ell_{i}\right)=\mathcal{N}\left(x ; m_{\mathrm{B}}^{(i)}, P_{\mathrm{B}}\right)$ with $m_{\mathrm{B}}^{(1)}=[0,500,0,0]^{T}$, $m_{\mathrm{B}}^{(2)}=[433,-250,0,0]^{T}, m_{\mathrm{B}}^{(3)}=[-433,-250,0,0]^{T}$, and $P_{\mathrm{B}}=\sigma_{\mathrm{B}}^{2} I_{4}$ where $\sigma_{\mathrm{B}}=10$.

Given a parent state $\mathbf{x}=(x, \ell)$ at the current time $k$ and setting $M_{\ell}=1$, from (20), the set of spawn labels at the next time $k+1$ is defined as

$$
\mathbb{T}_{+}(\ell)=\{(\ell, k+1) \times\{1\}\}=\{(\ell, k+1,1)\} .
$$

Additionally, we set the probability of detection constant, i.e., $p_{\mathrm{T}} \triangleq p_{\mathrm{T}}(\mathbf{x} ; \varsigma)$. Then, the spawn model is a conditional LMB RFS with parameters $\left\{\left(p_{\mathrm{T}}, f_{\mathrm{T},+}(\cdot \mid \mathbf{x} ; \varsigma)\right): \varsigma \in \mathbb{T}_{+}(\ell)\right\}$ where $p_{\mathrm{T}}=0.01$ and $f_{\mathrm{T},+}(\cdot \mid x, \ell ; \varsigma)=\sum_{i=1}^{3} \mathcal{N}\left(\cdot ; F x+d_{\mathrm{T}}^{(i)}, Q_{\mathrm{T}}\right)$ with $Q_{\mathrm{T}}=\sigma_{\mathrm{T}}^{2} I_{4}, \sigma_{\mathrm{T}}=5$. Each $d_{\mathrm{T}}^{(i)}$ is configured such that a spawn track with zero velocity is generated at a distance of $70 \mathrm{~m}$ from a parent state $x_{k}$ and in a direction relative to the parent's bearing $\theta$, i.e.,

$$
d_{\mathrm{T}}^{(i)}=\left[70 \cos \left(\theta+\phi^{(i)}\right), 70 \sin \left(\theta+\phi^{(i)}\right),-\dot{p}_{x, k},-\dot{p}_{y, k}\right]^{T}
$$

where $\phi^{(1)}=-80 \mathrm{deg}, \phi^{(2)}=-90 \mathrm{deg}$, and $\phi^{(3)}=-100 \mathrm{deg}$.

The maximum number of GLMB filter components is capped at 1000 . Using the Gibbs sampler to randomly generate hypotheses, the probabilities of survival and detection are tempered with values set to $\breve{p}_{\mathrm{S}, k}=0.90 p_{\mathrm{S}, k}$ and $\breve{p}_{\mathrm{D}, k}=0.90 p_{\mathrm{D}, k}$, respectively. This induces the sampler to yield more track termination and miss detection hypotheses, which expedites the termination of truly dead tracks while reducing the occurrence of dropped tracks. For more details on tempering techniques, see [34]. The CPHD filter is configured with a Bernoulli spawn model following the presentation in [20] and is capped at 1000 Gaussian mixture components.

Results are presented for 100 Monte Carlo simulations. The mean and standard deviation of cardinality estimates over time are shown in Figs. 3 and 4, while mean Optimal Sub-Pattern Assignment (OSPA) [42] distances are shown in Fig. 5. Mean OSPA localization and cardinality components are shown in Fig. 6 


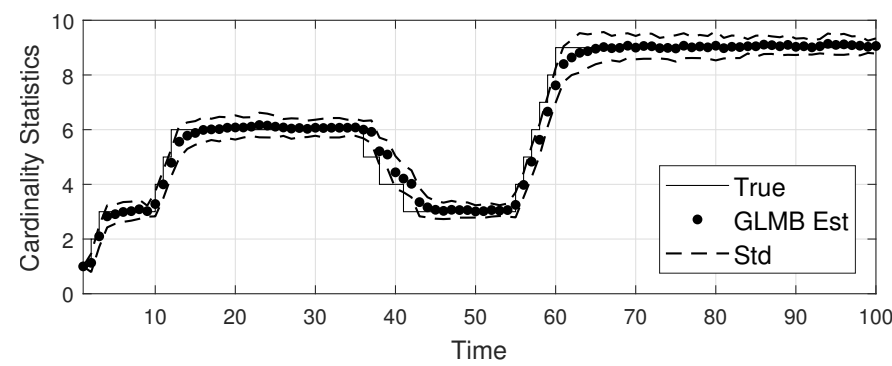

Fig. 3: Cardinality statistics for GLMB filter (100 Monte Carlo trials).

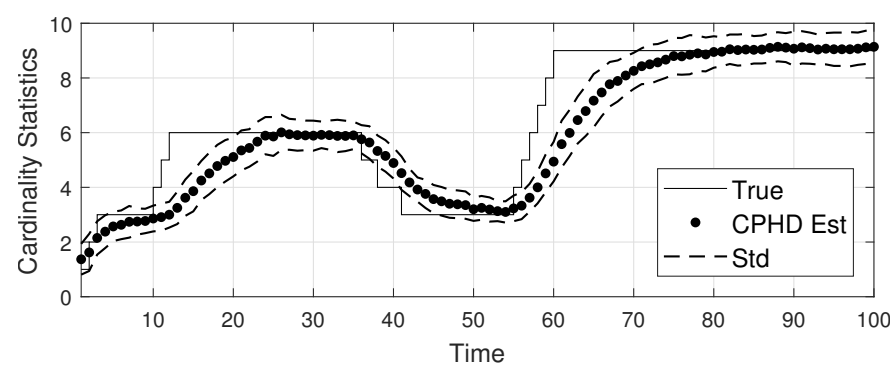

Fig. 4: Cardinality statistics for CPHD filter (100 Monte Carlo trials).

Similar to the results presented in [33], both filters accurately estimate cardinality with the GLMB filter providing a better cardinality variance estimate. The GLMB also exhibits better miss distance performance throughout the majority of the simulation. From Fig. 6 we see that the GLMB outperforms the CPHD in both cardinality and localization components overall. Consistent with the assessment in [33], lower estimated cardinality variance promotes improved cardinality performance. Improved localization performance is attributed to the GLMB's ability to propagate the filtering density more accurately and its immunity to the "spooky-effect". The CPHD filter is susceptible to this effect where in the event of a miss detection the PHD mass shifts away from undetected components to detected ones, regardless of the distance between them [33], [43].

The proposed GLMB filter's ability to capture ancestry information is demonstrated in Fig. 7. For each Monte Carlo run, the final label estimates at time $k=100$ are compared to the set of true labels of the same time which are presented in Table I The true labels are presented horizontally and are enumerated by the far left column. Shading is used to divide labels 1-6 into segments correlating with birth and spawning events in Fig. 7. where red, green, and yellow represent a birth track from which spawn originate, first generation spawn, and second generation spawn, respectively. Labels 7-9 are not considered for this analysis since they have no ancestry.

When comparing the final label estimates at time $k=100$ of each Monte Carlo run with the truth, estimated labels are segregated by common ancestry, e.g., from Table [1] labels 1 and 4 belong to one group, labels 2 and 5 belong to another, etc. For a given label group, the time of birth for their common birth ancestor track may differ from the truth, therefore it is necessary to trace the common ancestor track's label and state estimates toward the beginning of the given Monte Carlo run. Comparing this time history of state estimates with the truth, the originating birth region is determined. Then, the

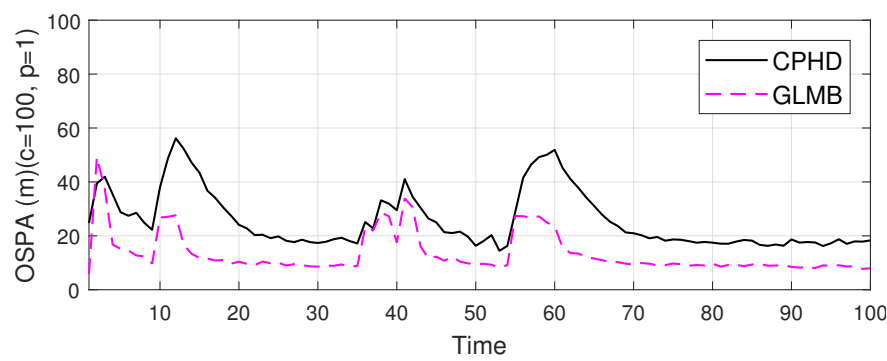

Fig. 5: OSPA distance for GLMB and CPHD filters (100 Monte Carlo trials).
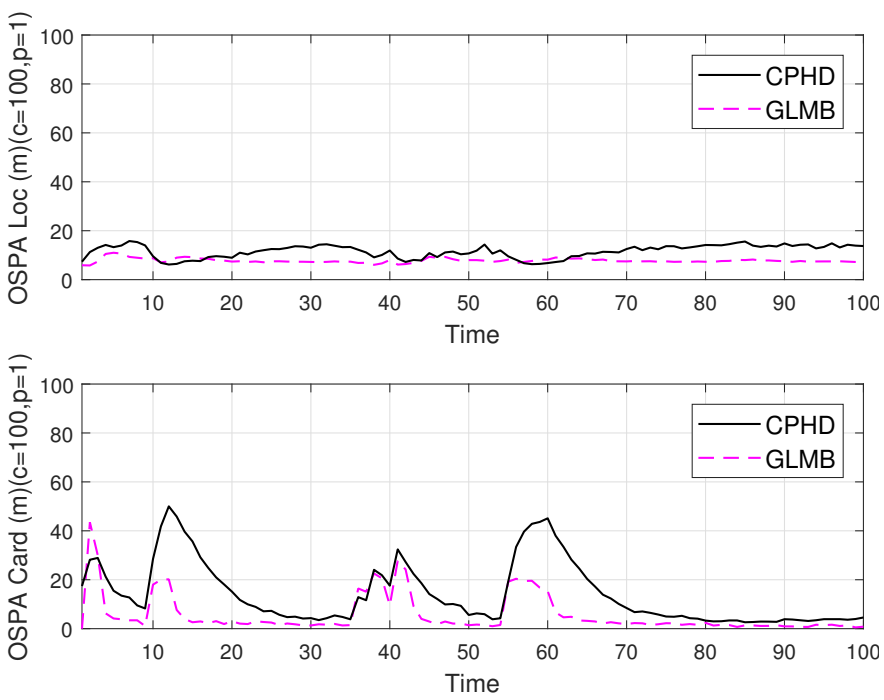

Fig. 6: OSPA components for GLMB and CPHD filters (100 Monte Carlo trials).

birth track's time of death is found by tracing its label from the beginning of the given run to the point in time when its label is no longer present in the set of label estimates. The remaining event times represented in Fig. 7, i.e., times of birth and spawning, are extracted from the labels of a final label estimates at time $k=100$ of the given Monte Carlo run.

TABLE I: Label Ground Truth at Time $k=100$

\begin{tabular}{ccccccc}
\hline Label \# & \multicolumn{7}{c}{ Label } \\
\hline 1 & 1 & 1 & 10 & 1 & 56 & 1 \\
2 & 2 & 2 & 11 & 1 & 58 & 1 \\
3 & 3 & 3 & 12 & 1 & 60 & 1 \\
4 & 1 & 1 & 10 & 1 & & \\
5 & 2 & 2 & 11 & 1 & & \\
6 & 3 & 3 & 12 & 1 & & \\
7 & 55 & 3 & & & & \\
8 & 57 & 1 & & & & \\
9 & 59 & 2 & & & & \\
\hline
\end{tabular}

The formats of Figs. 7(a) 7(c) are generally the same. Each birth region's true ancestry tree is at the far left and is aligned with a gridded area to the right with markers indicating GLMB filter estimated birth, death, and spawn times for 100 Monte Carlo runs. Each figure corresponds to one of the three modeled birth regions as indicated by the true track labels. We see from Fig. 7 that the GLMB filter accurately captures ancestry information overall, where the results in 


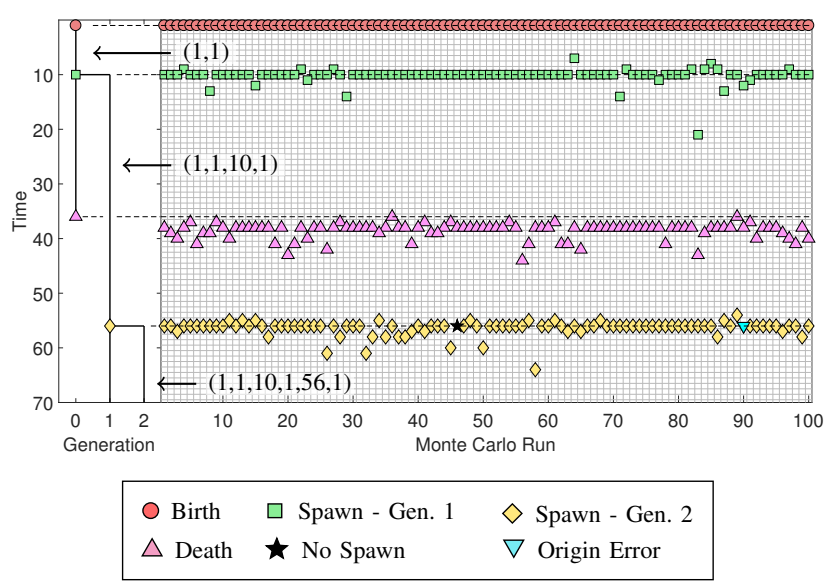

(a) Birth Region 1

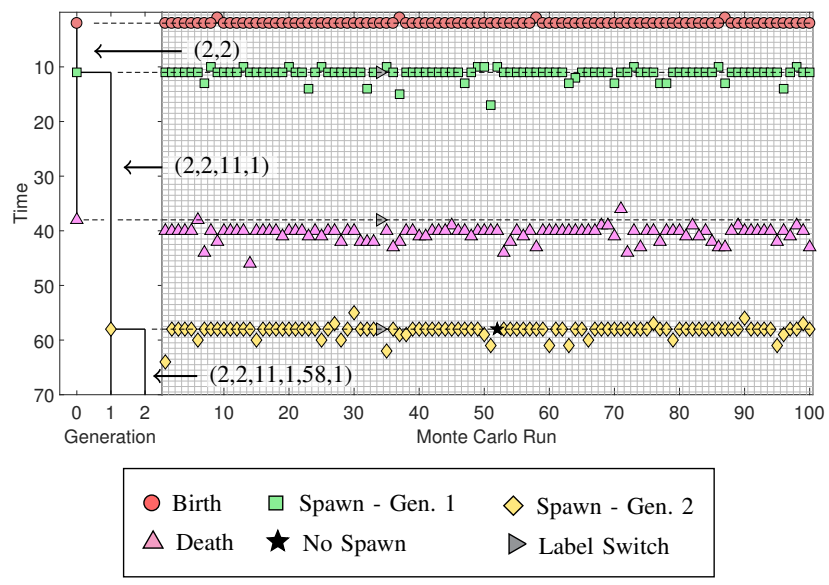

(b) Birth Region 2

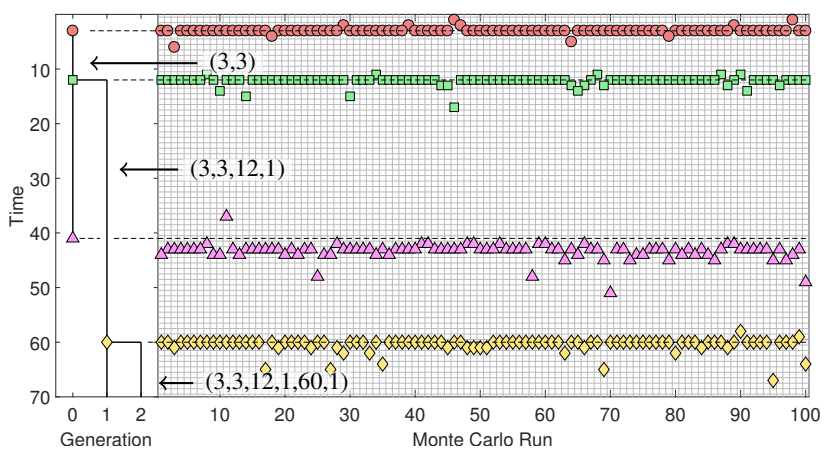

O Birth $\square$ Spawn - Gen. $1 \quad \triangle$ Death $\diamond$ Spawn - Gen. 2

(c) Birth Region 3

Fig. 7: Birth region ancestry truth and estimates. Each region's true ancestry tree is at the far left. A red circle at the top indicates birth, while the first and second generation spawn times are denoted by a green square and yellow diamond, respectively. The birth track's time of death is marked by a pink diamond. Markers within the gridded area indicate GLMB filter estimated birth, death, and spawn times for 100 Monte Carlo runs.

Fig. 7(c) exhibit the best performance. Fig. 7(a) indicates that the second generation spawn track $(1,1,10,1,56,1)$ was not spawned during run 46 and that the same generation track was estimated as originating from a different parent during run 90. In this specific case, track $(1,1,10,1)$ dropped prior to tracks crossing at the origin (see Fig. 2) and was later estimated as having spawned from one of the remaining first generation spawn tracks, subsequently spawning an object at time $k=56$. Similarly, Fig. 7(b) shows that track $(2,2,11,1,58,1)$ did not spawn during run 52 , while during run 34 , a label switch occurred when track $(2,2)$ essentially took the place of track $(2,2,11,1)$, going on to spawn track $(2,2,58,1)$ at time $k=58$. These inaccurate ancestry estimates are due to missed detections of either the parent track, spawn track, or both.

\section{CONClusion}

This paper presented the first GLMB filter to consider object spawning. Using a top-down formulation, a general labeled RFS density characterizing the predicted multi-object density of surviving, birth and spawn objects was derived. A joint prediction-update was performed yielding a density that was then approximated to form a posterior GLMB density while preserving its cardinality and PHD. A key innovation of the proposed filter is the capacity of spawn track labels to encapsulate their ancestry. The filter's ability to instantiate new tracks originating from previously known objects was verified by simulation. Our results can potentially be extended to accommodate measurement-based birth and spawn models.

\section{APPENDIX}

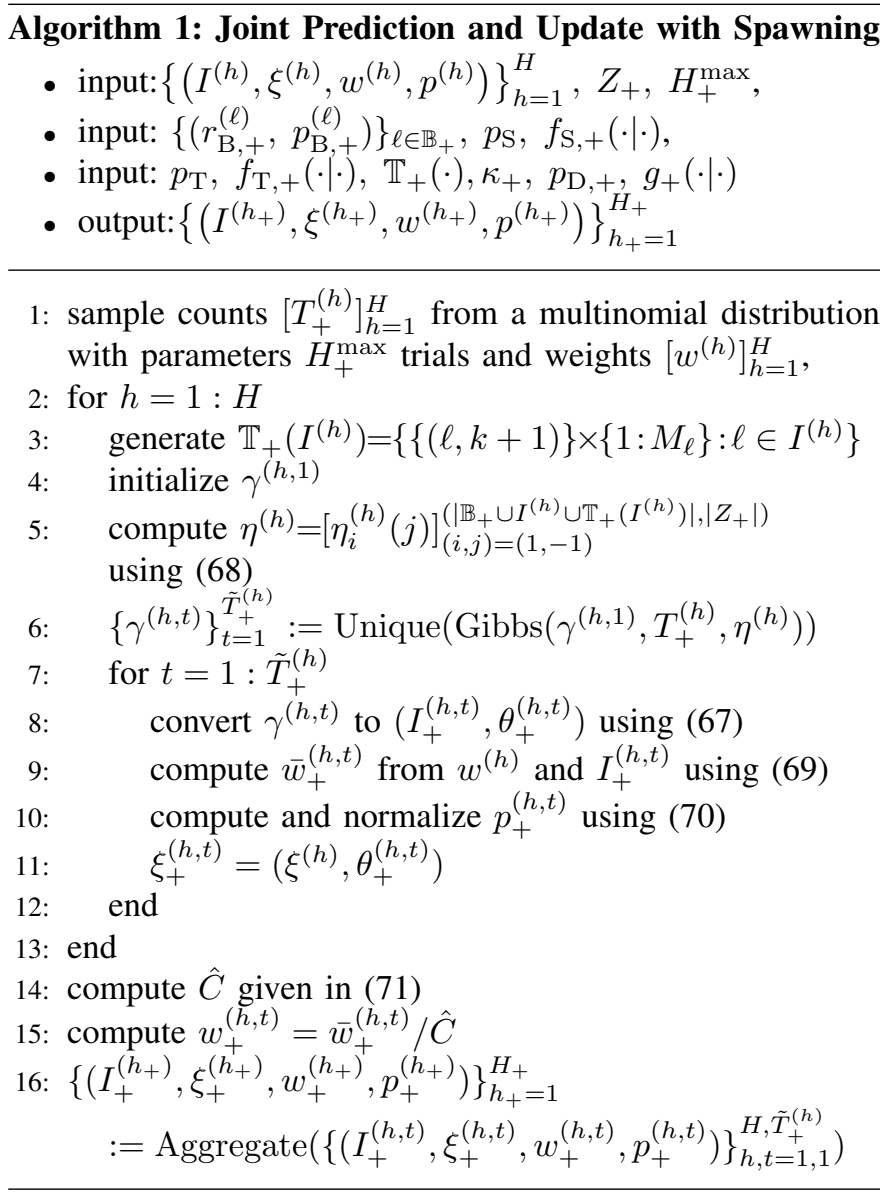




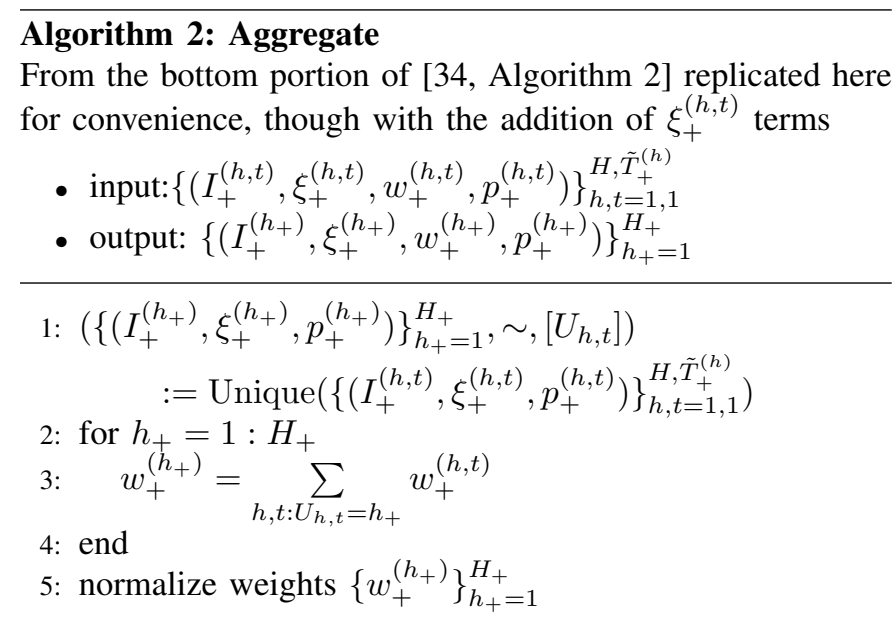

\section{REFERENCES}

[1] A. Munir and D. P. Atherton, "Maneuvring target tracking using different turn rate models in the interacting multiple model algorithm," in Decis. Control. 1995., Proc. 34th IEEE Conf., vol. 3, Dec. 1995, pp. 27472751.

[2] B. A. Jones, D. S. Bryant, B.-N. Vo, and B.-T. Vo, "Challenges of multi-target tracking for space situational awareness," 18th Int. Conf. Inf. Fusion, pp. 1278-1285, 2015.

[3] M. Hecker, "Expert system for processing uncorrelated satellite tracks," J. Guid. Control. Dyn., vol. 18, no. 5, pp. 1139-1144, 1995.

[4] P. W. Schumacher Jr, C. Sabol, A. Segerman, A. Hoskins, S. Coffey, K. Borelli, J. Addison, K. Hill, K. Roe, and B. Duncan, "Search and Determine Integrated Environment (SADIE) for automated processing of space surveillance observations," in AIAA/AAS Astrodyn. Conf. AIAA, vol. 4165, 2014, pp. 4-7.

[5] S. Blackman and R. Popoli, Design and Analysis of Modern Tracking Systems. Norwood, MA: Artech House, 1999.

[6] J. Mullane, B.-N. Vo, M. D. Adams, and B.-T. Vo, "A random-finite-set approach to Bayesian SLAM," IEEE Trans. Robot., vol. 27, no. 2, pp. 268-282, Apr. 2011.

[7] C. S. Lee, D. E. Clark, and J. Salvi, "SLAM with dynamic targets via single-cluster PHD filtering," IEEE J. Sel. Top. Signal Process., vol. 7, no. 3, pp. 543-552, 2013.

[8] E. Meijering, O. Dzyubachyk, and I. Smal, "Methods for cell and particle tracking," in Methods Enzymol., P. M. Conn, Ed. Academic Press, 2012, vol. 504, no. 9, pp. 183-200.

[9] R. Hoseinnezhad, B.-N. Vo, B.-T. Vo, and D. Suter, "Visual tracking of numerous targets via multi-Bernoulli filtering of image data," Pattern Recognit., vol. 45, no. 10, pp. 3625-3635, Oct. 2012.

[10] D. Reid, "An algorithm for tracking multiple targets," IEEE Trans. Automat. Contr., vol. 24, no. 6, pp. 843-854, 1979.

[11] T. Kurien, "Issues in the design of practical multitarget tracking algorithms," Multitarget-Multisensor Track. Adv. Appl., vol. 1, pp. 43-83, 1990.

[12] S. Blackman, "Multiple hypothesis tracking for multiple target tracking," IEEE Aerosp. Electron. Syst. Mag., vol. 19, no. 1, pp. 5-18, Jan. 2004.

[13] M. Mallick, S. Coraluppi, and C. Carthel, "Multitarget tracking using multiple hypothesis tracking," in Integr. Tracking, Classif. Sens. Manag. Theory Appl., M. Mallick, V. Krishnamurthy, and B.-N. Vo, Eds. Hoboken, New Jersey: Wiley-IEEE Press, 2012, pp. 165-201.

[14] Y. Bar-Shalom, Tracking and Data Fusion: A Handbook of Algorithms. Bloomfield, CT, USA: YBS Publishing, 2011.

[15] R. Mahler, Statistical Multisource-Multitarget Information Fusion. Norwood, MA: Artech House, 2007.

[16] - Advances in Statistical Multisource-Multitarget Information Fusion. Norwood, MA: Artech House, 2014.

[17] _ , "Multitarget Bayes filtering via first-order multitarget moments," IEEE Trans. Aerosp. Electron. Syst., vol. 39, no. 4, pp. 1152-1178, 2003.

[18] _ _ "PHD filters of higher order in target number," IEEE Trans. Aerosp. Electron. Syst., vol. 43, no. 4, pp. 1523-1543, 2007.

[19] M. Lundgren, L. Svensson, and L. Hammarstrand, "A CPHD filter for tracking with spawning models," IEEE J. Sel. Top. Signal Process., vol. 7, no. 3, pp. 496-507, 2013.
[20] D. S. Bryant, E. D. Delande, S. Gehly, J. Houssineau, D. E. Clark, and B. A. Jones, "The CPHD filter with target spawning," IEEE Trans. Signal Process., vol. 65, no. 5, pp. 13 124-13 138, Mar. 2017.

[21] K. Li, E. D. Miller, M. Chen, T. Kanade, L. E. Weiss, and P. G. Campbell, "Cell population tracking and lineage construction with spatiotemporal context,” Med. Image Anal., vol. 12, no. 5, pp. 546-566, 2008.

[22] T. Becker, A. Madany, and Others, "Morphology-based features for adaptive mitosis detection of in vitro stem cell tracking data," Methods Inf. Med., vol. 51, no. 5, p. 449, 2012.

[23] R. M. Baker, M. E. Brasch, M. L. Manning, and J. H. Henderson, "Automated, contour-based tracking and analysis of cell behaviour over long time scales in environments of varying complexity and cell density," J. R. Soc. Interface, vol. 11, no. 97, 2014

[24] D. S. Bryant and B. A. Jones, "Fragmentation event tracking with the GM-CPHD filter," Adv. Astronaut. Sci. 2016 AAS/AIAA Spacefl. Mech. Meet., 2016.

[25] P. W. Schumacher Jr and N. S. Command, "Tracking satellite break-ups," in US-Russian 2nd Sp. Surveill. Work. Conf. Proc. DTIC Document, 1996, pp. 174-182.

[26] K. J. DeMars and J. S. McCabe, "Tracking of the LANDSAT 2 rocket body primary breakup," in AAS/AIAA Spacefl. Mech. Meet., no. AAS:15420, Williamsburg, VA, Jan. 2015.

[27] “Space-Track website," 2017. [Online]. Available: https://www. space-track.org/documentation $\{\%\} 5 \mathrm{C}\{\#\} / \mathrm{faq}$

[28] B. Ristic, D. Clark, B.-N. Vo, and B.-T. Vo, "Adaptive target birth intensity for PHD and CPHD filters," IEEE Trans. Aerosp. Electron. Syst., vol. 48, no. 2, pp. 1656-1668, Apr. 2012.

[29] V. Maroulas and R. Mahler, "Tracking spawning objects," Radar, Sonar Navig. IET, vol. 7, no. 3, pp. 321-331, 2013.

[30] B.-N. Vo, B.-T. Vo, N.-T. T. Pham, and D. Suter, "Joint detection and estimation of multiple objects from image observations," IEEE Trans. Signal Process., vol. 58, no. 10, pp. 5129-5141, Oct. 2010.

[31] B.-T. Vo, B.-N. Vo, and A. Cantoni, "The cardinality balanced multitarget multi-Bernoulli filter and its implementations," IEEE Trans. Signal Process., vol. 57, no. 2, pp. 409-423, 2009.

[32] B.-T. Vo and B.-N. Vo, "Labeled random finite sets and multi-object conjugate priors," IEEE Trans. Signal Process., vol. 61, no. 13, pp. 3460-3475, Jul. 2013.

[33] B.-N. Vo, B.-T. Vo, and D. Phung, "Labeled random finite sets and the Bayes multi-target tracking filter," IEEE Trans. Aerosp. Electron. Syst., vol. 62, no. 24, pp. 6554-6567, Dec. 2014.

[34] B.-N. Vo, B.-T. Vo, and H. G. Hoang, "An efficient implementation of the generalized labeled multi-Bernoulli filter," IEEE Trans. Signal Process., vol. 65, no. 8, pp. 1975-1987, Apr. 2017.

[35] M. Beard, B.-T. Vo, B.-N. Vo, and S. Arulampalam, "Void probabilities and Cauchy-Schwarz divergence for generalized labeled multi-Bernoulli models," IEEE Trans. Signal Process., vol. 65, no. 19, pp. 5047-5061, Oct. 2017.

[36] F. Papi, B.-N. Vo, B.-T. Vo, C. Fantacci, and M. Beard, "Generalized labeled multi-Bernoulli approximation of multi-object densities," IEEE Trans. Signal Process., vol. 63, no. 20, pp. 5487-5497, Oct. 2015.

[37] B.-N. Vo, S. Singh, and A. Boucet, "Sequential Monte Carlo methods for multi-target filtering with random finite sets," IEEE Trans. Aerosp. Electron. Syst., vol. 41, no. 4, pp. 1224-1245, Oct. 2005.

[38] T. Vu, B.-N. Vo, and R. Evans, "A particle marginal Metropolis-Hastings multi-target tracker," IEEE Trans. Signal Process., vol. 62, no. 15, pp. 3953-3964, Aug. 2014.

[39] P. Craciun, M. Ortner, and J. Zerubia, "Joint detection and tracking of moving objects using spatio-temporal marked point processes," in 2015 IEEE Winter Conf. Appl. Comput. Vis. IEEE, Jan. 2015, pp. 177-184.

[40] F. Papi and D. Y. Kim, "A particle multi-target tracker for superpositional measurements using labeled random finite sets," IEEE Trans. Signal Process., vol. 63, no. 16, pp. 4348-4358, Aug. 2015.

[41] K. G. Murty, "An algorithm for ranking all the assignments in order of increasing cost," Oper. Res., vol. 16, no. 3, pp. 682-687, 1968.

[42] D. Schuhmacher and B.-N. Vo, "A consistent metric for performance evaluation of multi-object filters," IEEE Trans. Signal Process., vol. 56 , no. 8, pp. 3447-3457, 2008.

[43] D. Franken, M. Schmidt, and M. Ulmke, "'Spooky action at a distance" in the cardinalized probability hypothesis density filter," Aerosp. Electron. Syst. IEEE Trans., vol. 45, no. 4, pp. 1657-1664, Oct. 2009. 